\title{
Gradhiva
}

GRADHIV

Revue d'anthropologie et d'histoire des arts

13 | 2011

Pièges à voir, pièges à penser

\section{Le graphisme sur les corps amérindiens}

Des chimères abstraites?

Amerindian body art as abstract chimeras?

\section{Els Lagrou}

\section{CpenEdition}

\section{Journals}

Édition électronique

URL : http://journals.openedition.org/gradhiva/2040

DOI : 10.4000/gradhiva.2040

ISSN : 1760-849X

Éditeur

Musée du quai Branly Jacques Chirac

Édition imprimée

Date de publication : 18 mai 2011

Pagination : 68-93

ISBN : 978-2-35744-042-5

ISSN : 0764-8928

Référence électronique

Els Lagrou, "Le graphisme sur les corps amérindiens », Gradhiva [En ligne], 13 | 2011, mis en ligne le 18 mai 2014, consulté le 19 avril 2019. URL : http://journals.openedition.org/gradhiva/2040 ; DOI :

10.4000/gradhiva.2040

(c) musée du quai Branly 


\title{
Le graphisme sur les corps amérindiens
}

\author{
Des chimères abstraites?
}

Els Lagrou

\footnotetext{
-

1. J'ai conduit mon enquête de terrain chez les Cashinahua de l'aire indigène du Haut-Purus, dans l'État d'Acre au Brésil, durant dix-huit mois (entre 1989 et 1995). J'ai travaillé un mois en 2007 sur la traduction de chants rituels cashinahua avec Leôncio Salomão. La population cashinahua est estimée à environ sept mille personnes.
}

2. J'ai eu l'opportunité de discuter les idées développées dans cet article dans plusieurs séminaires et symposia organisés dans le cadre du projet Capes-Cofecub «Art-Image-Mémoire », à Paris et à Rio (entre 2007 et 2010), et je remercie particulièrement Carlo Severi, Anne-Christine Taylor, Denis Vidal, Aparecida Vilaça, Bruna Franchetto, Marco Antonio Gonçalves et Carlos Fausto pour leurs commentaires et suggestions.
J'explore dans cet article le rôle joué par le système graphique dans la vie perceptuelle des Indiens cashinahua de l'Amazonie brésilienne, en montrant comment un dessin abstrait opère le passage entre le visible et l'invisible dans un monde amérindien caractérisé par l'interchangeabilité des formes. Je suggère la possibilité d'étendre mes hypothèses à d'autres systèmes graphiques amérindiens apparentés ${ }^{1}$. Les images et les graphismes amérindiens sont des instruments perceptifs impliquant des opérations mentales qui présupposent une ontologie dans laquelle la transformabilité des formes et des corps occupe une place centrale. La reconnaissance du caractère perspectiviste (Viveiros de Castro 1996) et animiste (Descola 2005) des ontologies amérindiennes a mis en évidence l'importance de ce phénomène de transformabilité des formes, ainsi que celui d'un contraste constitutif entre intériorité et extériorité, sous-jacent au postulat qu'un être humain peut se cacher dans le corps d'un non-humain et vice versa. Ce constat a d'importantes conséquences pour l'étude des images matérielles et virtuelles produites par ces sociétés. Je démontrerai comment plusieurs procédés formels peuvent être vus comme des techniques perspectivistes, c'est-à-dire permettant au spectateur de changer de point de vue en fonction d'un «cadrage» spécifique (Bateson 1977).

Outre le contexte perspectiviste et animiste dans lequel se situe cette recherche, je prends comme point de départ la proposition de Carlo Severi de considérer le graphisme cashinahua comme une "chimère abstraite ${ }^{2}$ ". J'avais montré que ce graphisme induisait une focalisation de l'attention visant davantage à agencer et enchâsser le regard qu'à représenter des figures extérieures à lui-même. 


\section{PIÈGES À VOIR, PIÈGES À PENSER}

PAGE 68

Fig. 1 Panier kuki, le plus commun des paniers, utilisé par les femmes en général. Photo Eliane Camargo.

\footnotetext{
$\circ \circ$

3. Pour les Shipibo-Conibo voir Roe 1975, Gebhart-Sayer 1986, Illius 1987, Belaunde 2009. Pour les Asurini voir Müller 1990, pour les Wayãpi Gallois 2002, pour les Caduveo Lévi-Strauss 1955.
}

4. Sur les populations du Xingu, on se réfèrera à Barcelos Neto (2008) pour les Wauja, Franchetto (2003) pour les Kuikuro, Vidal (1992) pour les Kayapó-Xikrin.

5. L'analyse de la peinture de la Côte Nord-Ouest par Boas ( 1955 [1928]) et celle de la peinture des Caduveo par Lévi-Strauss [1955] préfigurent cette interprétation de la peinture corporelle. Les deux auteurs ont perçu la relation intrinsèque entre corps et graphisme. Lévi-Strauss relate le cas d'une femme caduveo qui, pour représenter la peinture faciale sur papier, dessina un visage en forme de cœur, comme si pour représenter le graphisme sur une surface, on devait scalper la face et l'étirer. Boas a montré qu'on peut voir les couvertures héraldiques de la Côte Nord-Ouest comme des «animaux disséqués 》. Quand on rejoint les parties du corps sur une boîte, on obtient un corps couvert par une peau.
J'avais également signalé son dynamisme : quoique "abstrait», il peut ouvrir à la perception d'une figuration virtuelle, image mentale qui n'est pas explorée par Warburg et Severi pour les images figuratives (Severi 2007).

Je chercherai ici à explorer les implications de l'association entre les concepts de "chimère " et d'" abstraction" chez les "peuples à dessin", comme sont souvent désignés en Amazonie occidentale, en sus des Cashinahua, les Shipibo, les Conibo, les Marubo (groupes de langue pano) et les Piro (de langue arawak). Cette catégorisation pourrait être étendue, pour des raisons d'affinité stylistique et de signification générale du système graphique, à d'autres ethnies productrices de "systèmes complexes de dessins" (complex design systems, Gow 1989), comme les Tupi Yudjá (Juruna), les Asurini et Wayãpi, ainsi que les Caduveo, de langue guaikuru?. Les populations du complexe culturel du Haut-Xingu utilisent également des peintures qui couvrent le corps entier avec des motifs entrelacés, tout comme les Kayapó 4 . Cet ensemble de peintures corporelles contraste avec ceux qui produisent des "marques", des dessins isolés qui ne couvrent pas la surface entière du support, comme c'est le cas en Amazonie occidentale chez les Ashaninka, les Achuar, les Culina et tant d'autres (Taylor 2003; Beysen 2008).

\section{Usages rituels du graphisme chez les Cashinahua}

L'étude de l'utilisation du graphisme dans deux contextes rituels distincts m'a permis de comprendre la nature de l'efficience du kene - le motif géométrique - en tant qu'instrument de transformation (Lagrou 2007a, 2009b). Le premier de ces contextes est celui du rite de passage dont la fonction est de remodeler le corps des enfants et dans lequel le dessin exerce un rôle important, puisqu'il fait de la peau un médiateur entre l'extérieur et l'intérieur du corps. Le second contexte rituel est celui de l'ingestion d'ayahuasca (une boisson psychotrope) par les chasseurs, dans lequel le dessin mental, virtuel, est évoqué dans le chant et visualisé par le participant; il fait office de médiation entre l'espace perceptible du quotidien et l'espace visionnaire de l'ayahuasca.

Dans les deux cas, le graphisme est perçu dans sa relation avec un support. Dans le premier, le dessin sur les corps des jeunes en cours de «fabrication » augmente la perméabilité de la peau et ouvre donc le corps à l'intervention rituelle. Dans le second cas, le dessin opère le passage d'une perception par l'œil à une perception virtuelle. "Il faut rester dans le dessin ", recommande-t-on aux néophytes pour qu'ils ne se perdent pas dans leur vision. On dit aussi que "chaque dessin s'ouvre sur la révélation d'une figure (dami) et plus tard d'un esprit (yuxin) ».

Voyons d'abord le contexte d'utilisation rituelle du dessin. Certaines peintures rituelles tachetées équivalent à couvrir le corps humain d'une peau animale, transformant provisoirement, comme dans les rituels de masques, les hommes en jaguar ou en anaconda. Cette utilisation de la peinture s'observe chez les Cashinahua à l'occasion des rites de fertilité, où les taches de roucou appliquées sur les visages et les corps servent à différencier les moitiés, l'une adoptant le dessin du jaguar (inu keneya) et l'autre du puma (txaxu inu). Cette peinture s'appelle dami, "transformer ", "masquer ", et diffère radicalement du kene kuin, dessin ou graphisme. Le graphisme (kene) au genipa ne masque pas, ne transforme pas l'identité de son porteur, il le fabriques. Lévi-Strauss qualifie ce genre de graphisme de "seconde peau ", une peau sociale; il considère que toutes les peintures corporelles amérindiennes obéissent à la logique du masque. Il me semble pourtant que 
l'on a affaire ici à deux phénomènes différents : l'un relève de la fabrication d'un corps spécifique, cashinahua, et l'autre relève des technologies d'« altérisation " qui sont employées pendant les rituels.

Les taches de roucou, associées à des vêtements de feuilles et parfois à des masques, transforment la personne en esprit de la forêt se préparant à envahir le village voisin. L'utilisation de l'« habit de l'Inca " (inkan tadi) pendant le rituel txidin suit la même logique : il transforme le spécialiste le temps du rituel en Inka, ennemi prototypique, dieu de la mort et destin futur des humains. Vêtements, peintures et feuilles constituent tous des agencements de perspectives autres. L'usage rituel réplique ainsi des épisodes mythiques où les hommes se sont transformés en animaux en se peignant ou en couvrant leur corps, chaque type de peinture renvoyant à une espèce différente (Capistrano de Abreu 1941).

L'utilisation de la peinture au genipa pendant le rite de passage suit, en revanche, une logique opposée, celle de la " fabrication » d'un corps plutôt que sa métamorphose. Pour que l'intervention rituelle soit efficace, le corps doit être peint. Comme chez les Gê, différents graphismes accompagnent les étapes de la transformation corporelle. Toutefois, chez les Cashinahua ces différences ne se manifestent pas, comme chez les Kayapó, au moyen des motifs employés : tous les dessins viennent de la même peau de l'anaconda/boa et sont utilisés par les deux sexes et à tous les âges (à l'exception de la petite enfance). L'écart entre les étapes est marqué par des différences dans le style d'exécution utilisé. Alors qu'elle est fine et précise chez les initiés et les adultes, la peinture des enfants qui passent par le rite de passage doit être grosse et «maladroite» (tube kene), pour que

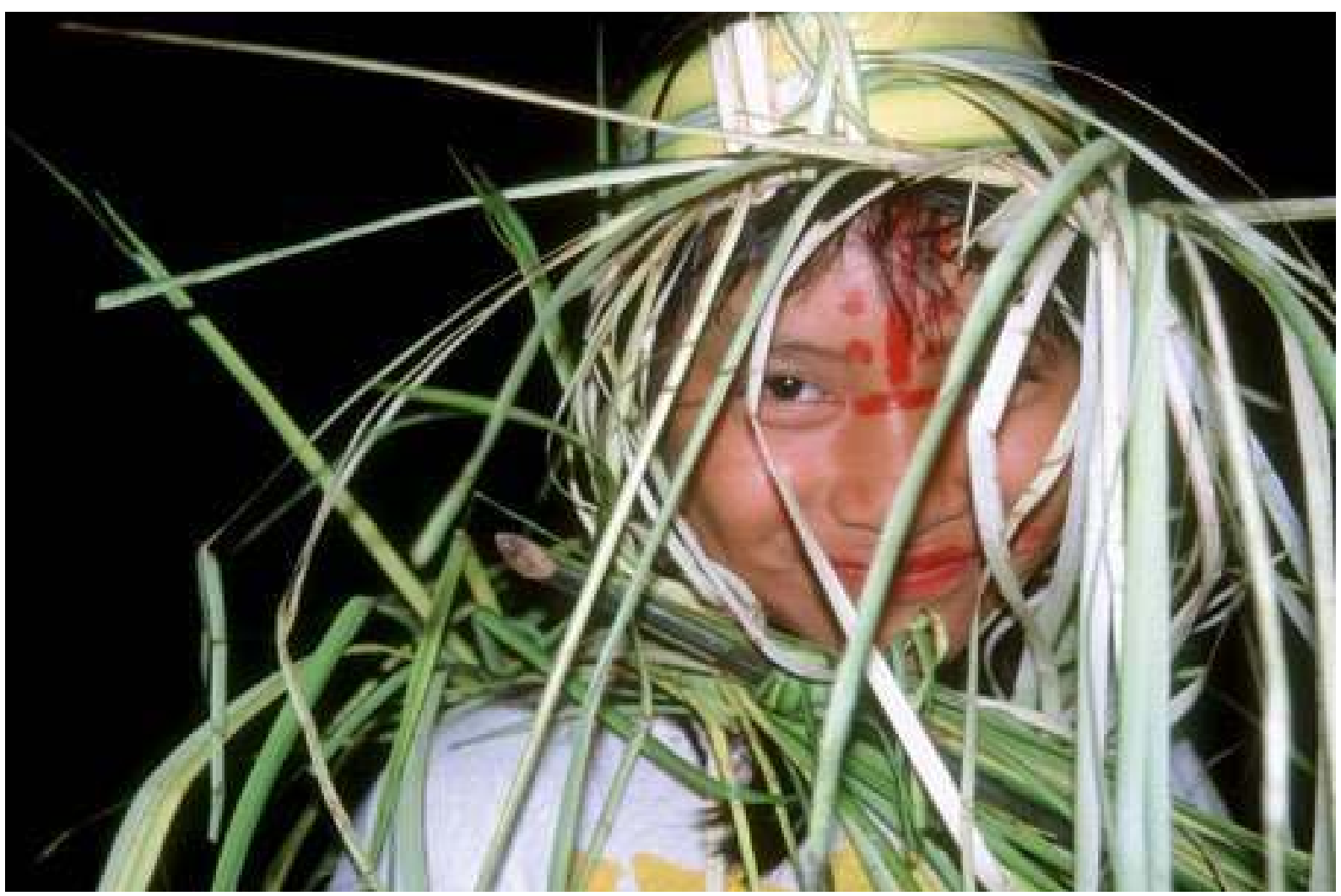

Fig. 2 Jeune fille déguisée en esprit de la forêt, 1995. Photo Els Lagrou. 


\section{PIÈGES À VOIR, PIÈGES À PENSER}

-

6. Si pour les Cashinahua la peinture participe dans le processus de transformation, chez les Piro et les Xinguaniens le corps en réclusion, en phase de fabrication, est nu. Selon Gow, la peinture corporelle des Piro est considérée comme résultant des «pensées des femmes». Leurs dessins se suffisent à eux-mêmes, par leur beauté compulsive qui focalise l'attention sur la perfection des formes de la jeune femme récemment sortie de réclusion. D'autre part, les humains partagent avec le jaguar et l'anaconda la qualité d'《être à dessin ». Le résultat paradoxal de l'art des femmes est de produire un être dont la beauté ressemble à celle - effrayante - du jaguar. Les Piro ne se peignent pas dans la vie quotidienne, lorsqu'ils sont entre corésidents, mais seulement pour rencontrer ceux avec lesquels on ne vit pas bien (Gow 2001 : 103-129). II existe un phénomène similaire dans le Haut-Xingu, où le graphisme marque également l'étape finale de la fabrication du corps (un processus occulté aux yeux du public] ainsi que la rencontre avec un alter distant qu'on veut impressionner et intimider par une beauté féroce.

7. Ingold propose une approche comparative des multiples sens du mot « ligne » et suggère que la relation entre ligne tracée et ligne tissée est présente dans l'histoire de l'Occident prémoderne ainsi que dans des contextes culturels non occidentaux renvoyant au mouvement des corps (Ingold 2007). Toutefois, ce qui nous intéresse ici est un autre genre de mouvement, celui de la perception mentale qui opère le passage entre ce qui est visible et ce qui ne l'est pas encore. l'action rituelle, les chants, les prières et les bains médicinaux puissent mieux pénétrer leur peau. Le dessin aux larges traits semble lever l'opacité du corps. Les nourrissons, par contraste, auront tout leur corps couvert de genipa pour le "fermer ", le protéger contre les influences extérieures ${ }^{6}$ (fig. 3).

Ces différents usages de la peinture nous permettent de voir la peau peinte comme un tissu, comme un panier : c'est l'organe de contact et de passage entre l'intérieur et l'extérieur, entre contenant et contenu. C'est la peau qui rend possible la création d'un " corps " susceptible de cacher en soi une intériorité. Dans son introduction à Wrapping in images, Alfred Gell (1993) propose des réflexions intéressantes sur ce qui pourrait constituer une anthropologie de la peau, en liant notamment la peau au placenta. Cette idée est présente aussi chez Peter Gow $(1999,2001)$, quand il note que pour les Piro le placenta, dont le réseau veineux est particulièrement saillant, est considéré comme le "premier dessin ». Pour que le nouveau-né survive, il faut que ce "premier dessin", désigné aussi comme «son jumeau ", soit enterré. L'enfant lui-même reste alors temporairement sans dessin, jusqu'à ce que l'art des femmes lui en rende un. Chez les Cashinahua, " pour que l'enfant puisse naître facilement» le chant rituel mentionne ses «vieux vêtements qui brûlent». Tout indique que ces "vêtements", qu'il doit échanger contre un tissu nouveau dans lequel il sera enveloppé, correspondent au placenta (Lagrou 1998a, 1998b).

On peut engager cette anthropologie de la peau dans une voie que Gell et Gow n'ont pas explorée, en analysant conjointement la fabrication des objets et des personnes. Ainsi Lúcia van Velthem (2003) étudie le parallèle entre artefacts et corps en montrant comment les Wayana utilisent les mêmes termes techniques pour la production et la décoration des artefacts et des humains. Le mythe d'origine de la femme wayana montre qu'elle est fabriquée de telle sorte qu'elle puisse agir : la première tentative de fabrication révéla que l'argile était trop lourde, et que la cire fondait sous la chaleur du soleil. Ce fut finalement la femme panier qui se révéla apte à exécuter les activités féminines. Le corps de la femme wayana fut donc tressé, comme le fut le corps du boa chez les Cashinahua et les Wauja. Les Asurini apprirent d'abord à produire les dessins par le tressage (Müller 1990). L'association entre le tressage, le tissage, le dessin et le corps de l'anaconda est extrêmement récurrente en Amazonie7 (Barcelos Neto 2008 : 231; Lagrou 1991, 1996).

Chez les Cashinahua, c'est aussi un mythe d'origine qui traduit l'idée que la peau est tissée. L'origine de l'anaconda/boa, maitre des dessins et de toutes les images virtuelles possibles, maitre aussi du flux du sang, se situe dans le mythe du grand déluge. Plusieurs artefacts se transforment à cette occasion en animaux, le panier aux dessins devenant la tête de l'anaconda, la massue devenant le gymnote, et un couple d'amants couché dans leur hamac devenant l'anaconda. Dans l'intérieur de ce boa, être surnaturel androgyne, se trouvent les agentivités féminines et masculines combinées, sa peau étant un hamac aux motifs tissés. Pour les Cashinahua, le boa et l'anaconda sont des transformations l'un de l'autre. Quand il est jeune, le boa vit sur la terre pour enseigner aux hommes, quand il devient fatigué et âgé, il se retire dans l'eau où il règne comme un grand chef, un "président ». C'est l'esprit du boa qui a enseigné l'art du graphisme aux femmes (kene), alors que l'expérience de l'ayahuasca, monde d'images (dami) et d'esprits (yuxin), a été enseigné aux hommes par l'anaconda. L'ayahuasca (nawan huni) est le sang ou l'urine de l'anaconda (duanuan himi, dunuan isun), Yube, maître du monde aquatique. C'est dans ce second contexte rituel, d'ingestion d'ayahuasca, 


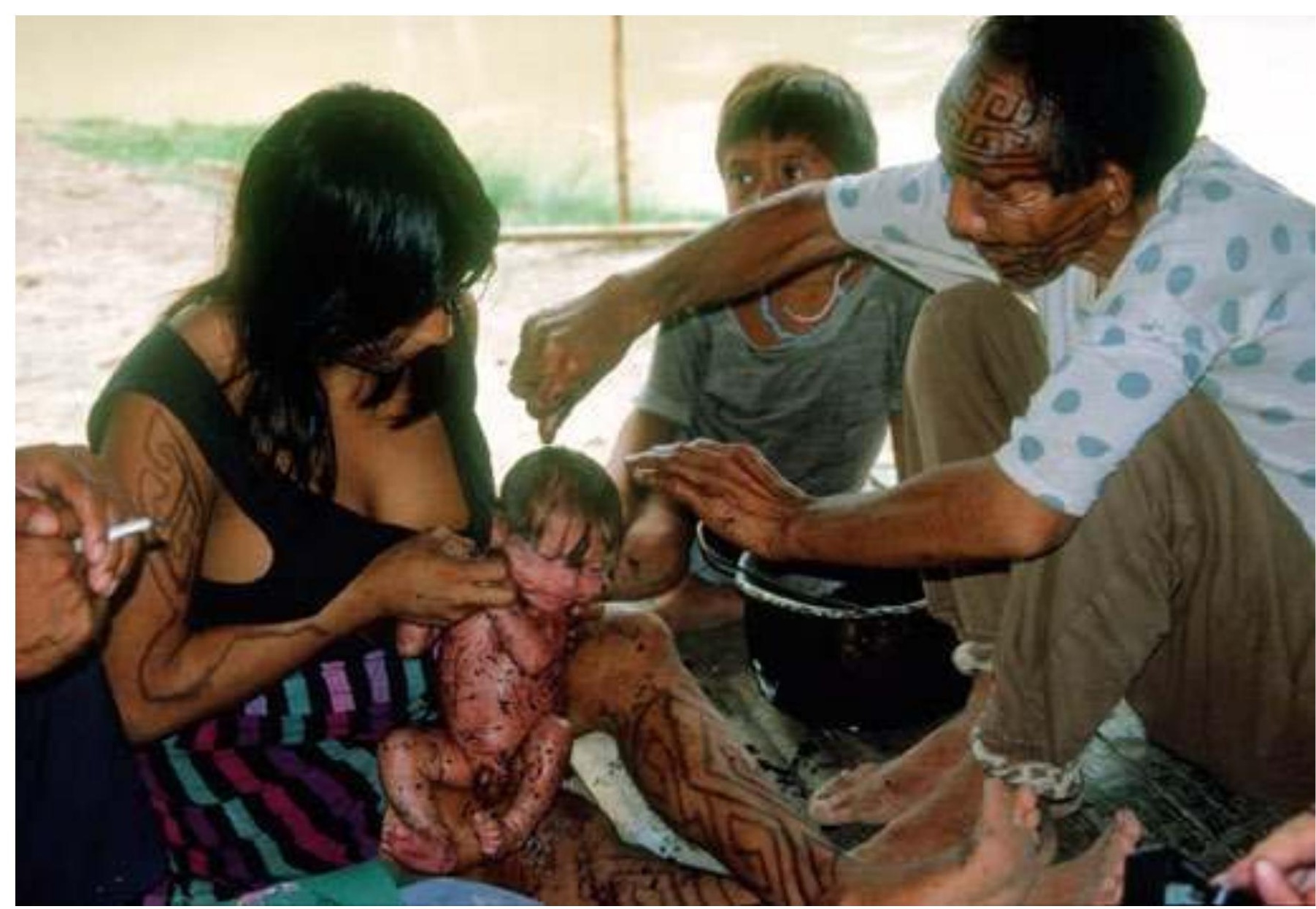

Fig. 3 Peinture du nourrisson. Photo Els Lagrou.

que nous pouvons examiner un discours explicite sur le passage de l'abstraction à la figuration dans la perception mentale des participants.

"Sur la peau de Yube on peut trouver tous les dessins possibles, il y en a vingt-cinq, mais chacun de ces dessins est à l'origine d'une multiplicité d'autres, car à la fin ils appartiennent tous à la même peau du boa. " (Agostinho Manduca Kaxinawa)

«Le dessin du boa contient le monde. Chaque tache sur sa peau peut s'ouvrir et montrer la porte d'entrée dans de nouvelles formes. La peau de Yube a vingt-cinq taches, qui sont les vingt-cinq dessins qui existent. » (Edivaldo Domingos Kaxinawa)

"Quand nous buvons son "sang", il nous montre tout ce qu'il a fait dans sa vie, son village, sa science. Yube se transforme en plusieurs choses, plusieurs serpents, plantes, lianes, en personne, eau, oiseau. Tous ses motifs peuvent se transformer en vision. Le kene est Yube qui se présente. Dami, les figures, c'est comme yuda baka - l'ombre du corps. Tu le vois, mais tu ne le retiens pas. Il s'en va après le nixi pae (la liane forte). C'est le dami - la transformation - du nixi pae du yuxibu (le maître). Il est mort, mais il n'est pas mort. Puisque son corps s'est transformé en liane. Yube est notre dieu. Il a laissé ce breuvage pour les siens, pour qu'ils ne pleurent plus, pour qu'il ne leur manque plus, puisqu'il est là, il se révèle. Ainsi comme ton fils verra tout ce que tu as fait dans ta vie, parce qu'il est sorti de dedans, la liane, quand elle est dans ton intérieur, te fait voir ce qui lui appartient. » (Agostinho Manduca Kaxinawa) 
Fig. 4 et 5 Motifs de tressage amazoniens. Photo Denise Andrade.

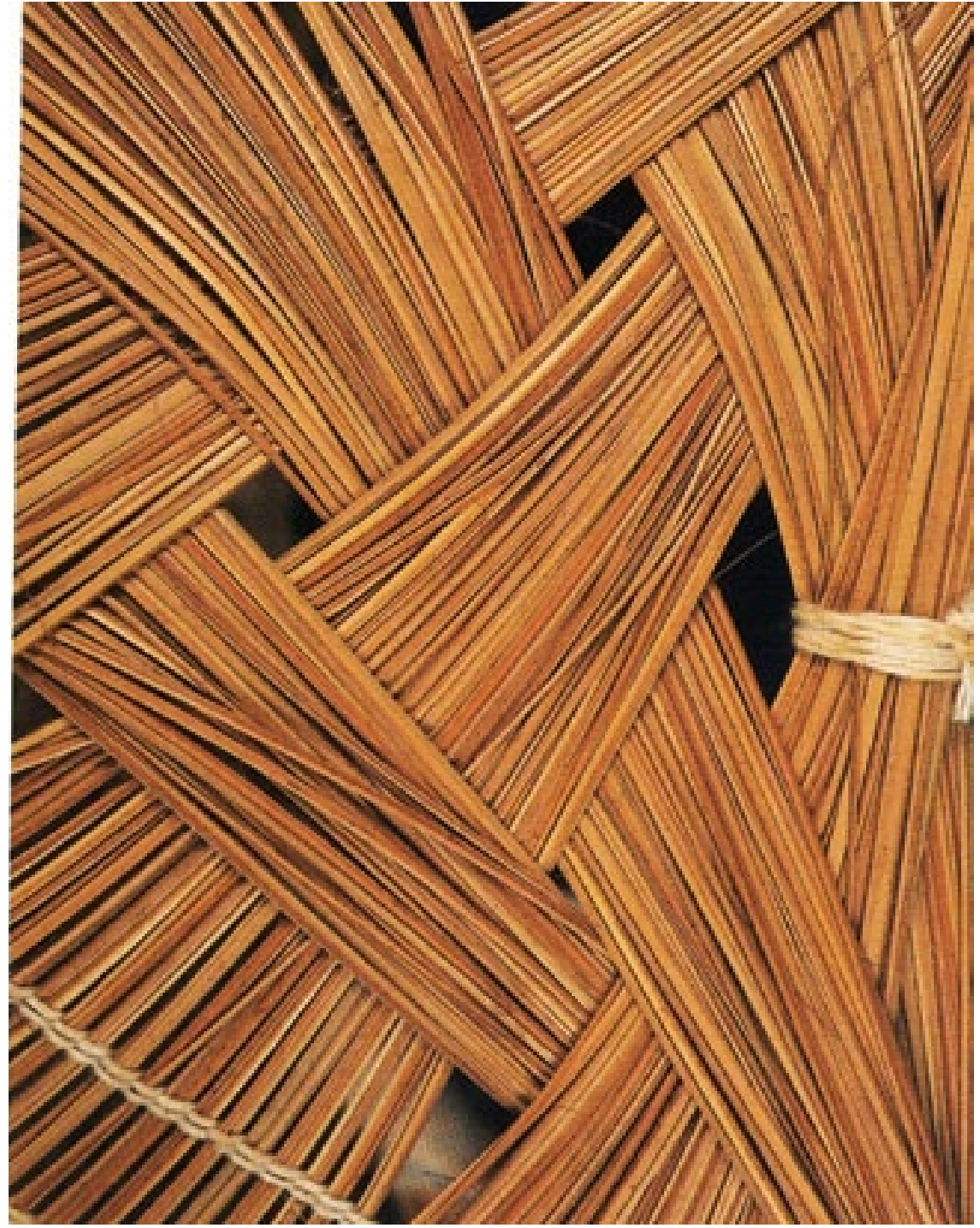

Ces citations font clairement référence au thème du passage du graphisme à la figuration virtuelle, à l'image mentale qui n'est pas donnée à voir dans le dessin mais qui peut être vue par ceux qui y sont préparés. Elles nous renvoient, en bref, au thème de la technique de la transformation visuelle. Les Cashinahua connaissent plusieurs techniques de médiation qui leur permettent de passer d'un point de vue à l'autre, et toutes ces techniques ont à voir avec des processus contrôlés de perception et de production des formes. Un chasseur, s'il veut être efficace en tant que chasseur, considère les singes comme yuinaka, du gibier. Les techniques de transformation de l'animal en viande passent par la chasse et par le feu, technique transformationnelle par excellence qui vise à le défigurer, l'effacer, le désubjectiver. Le gibier devient viande mais libère un double, son image. Et cette image se caractérise dès lors par son rapport instable avec le corps, puisqu'il lui en manque un, ce qui peut se traduire par des maladies chez les humains. Après avoir tué et/ou mangé un singe, le chasseur doit donc, pour rester en bonne santé, ingérer régulièrement de l'ayahuasca au cours du rituel du nixi pae. Il pourra alors identifier la cause de ses migraines : le double du singe venu danser sur sa tête. Le chant rituel identifie la cause du mal en même temps qu'il éloigne le double. L'esprit de l'œil du chasseur échappe ainsi au piège des singes. Selon Leôncio, grand spécia- 


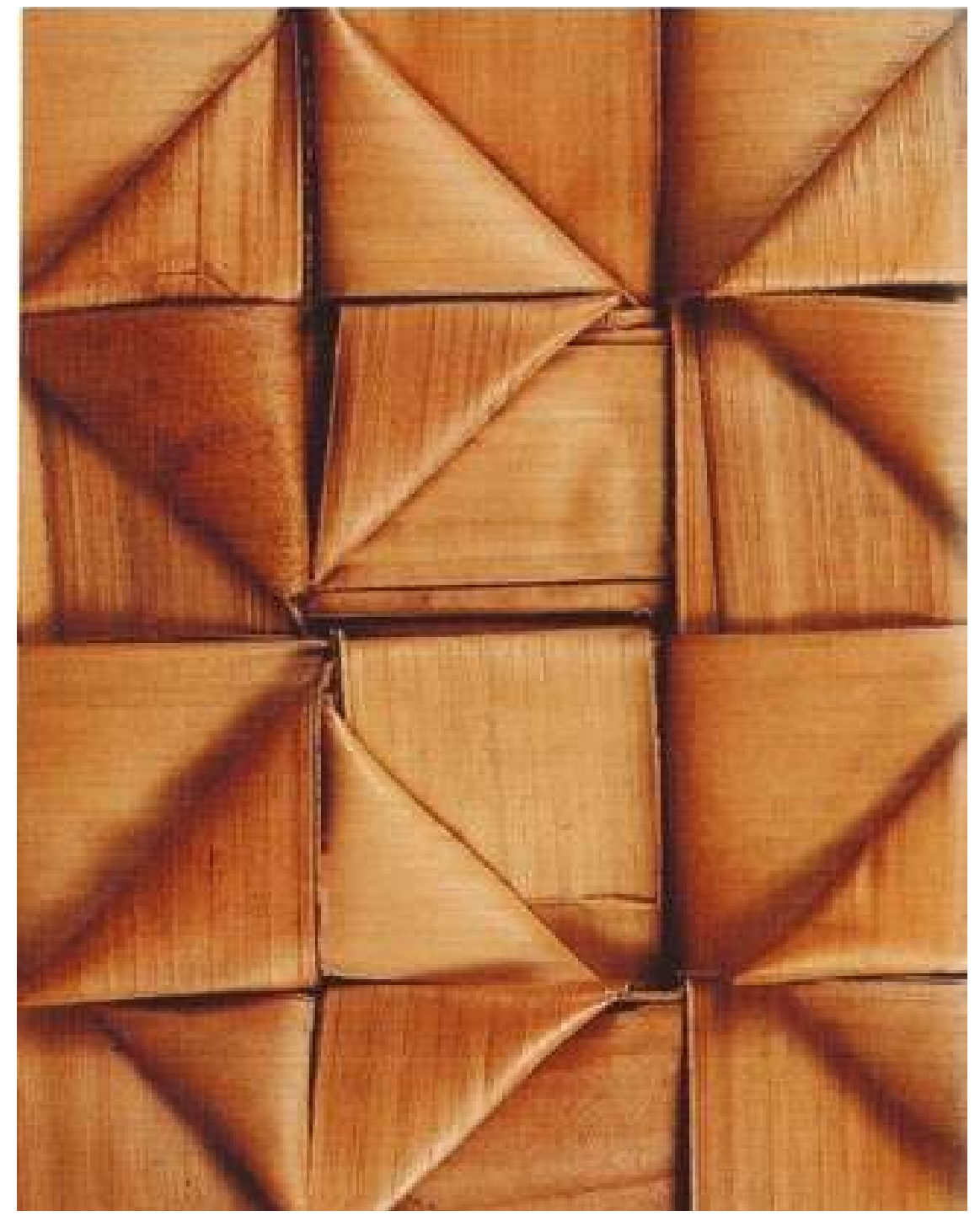

liste cashinahua de l'ayahuasca avec qui j'ai travaillé sur les chants d'ayahuasca, quand on prend le nixi pae, il faut chanter tout ce qu'on a ingéré, tout ce qu'on a mangé, les singes, les pécaris, mais aussi le tabac, le piment, et évidemment le nixi pae lui-même, c'est-à-dire la liane et la feuille à l'origine du breuvage.

Le lien entre substance et image est direct : on voit ce que l'on a ingéré. On devient partiellement ce que l'on mange. Les doubles des êtres qui ont été tués et mangés cherchent par vengeance à recouvrir les esprits de l'œil (bedu yuxin) de ceux qui les ont mangés avec leurs habits, tadi (dans le cas du pécari), leurs décorations corporelles, leurs colliers ou leurs dessins (dans le cas de Yube l'anaconda, le maitre de l'ayahuasca). Tous ces attributs sont des agencements du point de vue du double ingéré qui vient capturer l'esprit de l'agresseur pour l'emprisonner dans un nouveau corps, celui que le double a perdu. Ce que voit celui qui est sous l'effet de l'ayahuasca, ce n'est donc pas seulement l'autre sous la forme d'une humanité commune, mais sa propre intériorité - son esprit de l'œil - englobée par les habits, les peaux, les décorations de cet autre qu'il vient d'ingérer. Celui qui a été englobé par l'ingestion revient englober son agresseur par ses images. La relation entre images, substances, fluides et corps est ainsi hautement corrélative et transformationnelle. 


\section{La figuration des corps}

Les sociétés amérindiennes, surtout en Amazonie, produisent très peu de figuration. Si peu, que l'on pourrait se demander si les Amazoniens sont «iconophobes " (Taylor 2010; Lagrou 2005, 2007a). Je suggère que l'utilisation répandue de l'abstraction et l'évitement de la représentation figurative dans les expressions bidimensionelles amazoniennes s'explique par le fait que les motifs aident à constituer des surfaces qui contiennent des corps (ou sont appliqués sur de telles surfaces), et non pas qu'ils représentent des corps. Plusieurs mythes d'origine des systèmes graphiques amérindiens font coïncider l'apprentissage ou l'apparition des motifs graphiques avec la technique du tissage, ce qui suggère que le dessin est un élément constitutif de la fabrication de la peau ou de la surface des artefacts en général.

Ces systèmes graphiques coexistent toutefois avec un art figuratif souvent minimaliste, qui développe jusqu'à l'extrême la logique du «modèle réduit » de Lévi-Strauss (1964), comme on peut le noter dans l'anthropomorphisme et le zoomorphisme discrets des bancs xinguaniens et tukano, des poupées karajá, des vases shipibo, des effigies asurini et des maracas araweté, pour ne donner que quelques exemples. Ces artefacts sont tous considérés comme des quasi-corps, et les indices de ce caractère distinctif vont de détails extrêmement subtils jusqu'à la figuration complète.

Le tabouret utilisé par les jeunes Cashinahua est un exemple de cette logique selon laquelle les artefacts sont des corps en modèle réduit. Sa fabrication réplique celle du corps de l'enfant, qui est un artefact autant que le tabouret luimême. Le tabouret est désigné dans le chant rituel par la formule « deux jambes et un trou au milieu ", figurant la base du corps, ce qui le soutient. Il est fait des racines tubulaires de l'arbre lupuna, considéré comme «arbre de la vie » (Lagrou 2002b, 2007b); il est sculpté par le père, comme l'est le fœtus dans le ventre de sa mère; il est peint au genipa par la mère, comme le sera l'enfant pendant le rite de passage nixpu pima et après.

L'image synthétise les éléments minimaux qui caractérisent la manière d'agir du modèle et c'est pourquoi elle est un indice plutôt qu'un symbole ou une icône de son modèle (Gell 1998). Le tipiti (tube à manioc) des Wayana est un artefact qui partage avec le boa la capacité agentive de constriction, et c'est cette constriction qu'il doit utiliser pour transformer le manioc en pâte. Si le tipiti n'a ni queue ni tête, c'est pour qu'il ne puisse pas se transformer en boa, son modèle, un être indépendant dévorateur d'humains (Velthem 2003 : 130; Lagrou 2009a).

\section{Abstractionnisme}

Depuis la fin des années 1980, plusieurs spécialistes ont signalé l'importance d'une tendance à l'abstraction dans les graphismes amérindiens ${ }^{8}$, ce qui pose la question de leur référence. En s'éloignant d'une focalisation exclusive sur le rapport entre graphisme et communication verbale, ces auteurs ont perçu que le sens résidait souvent davantage dans les caractéristiques globales du style que dans les unités minimales de signification. Les précurseurs de cette appro-

8. Roe 1975; Gow 1989, 1999, 2001 ; Gebhart-Sayer 1986 ; Illius 1987 ; Guss 1989; Lagrou 1991, 1995, 1998a, 1998b, 2002a, 2005, 2007a, 2009b; Taylor 2003, 2010. che sont Lévi-Strauss $(1955,1958)$, dans ses écrits sur la peinture faciale caduveo, et Bateson (1977), dans son article sur les peintures balinaises. Bateson avait défendu l'idée que le message métacommunicatif d'un tableau, accessible par le style qui fait percevoir la nécessaire complémentarité entre les contraires, est 


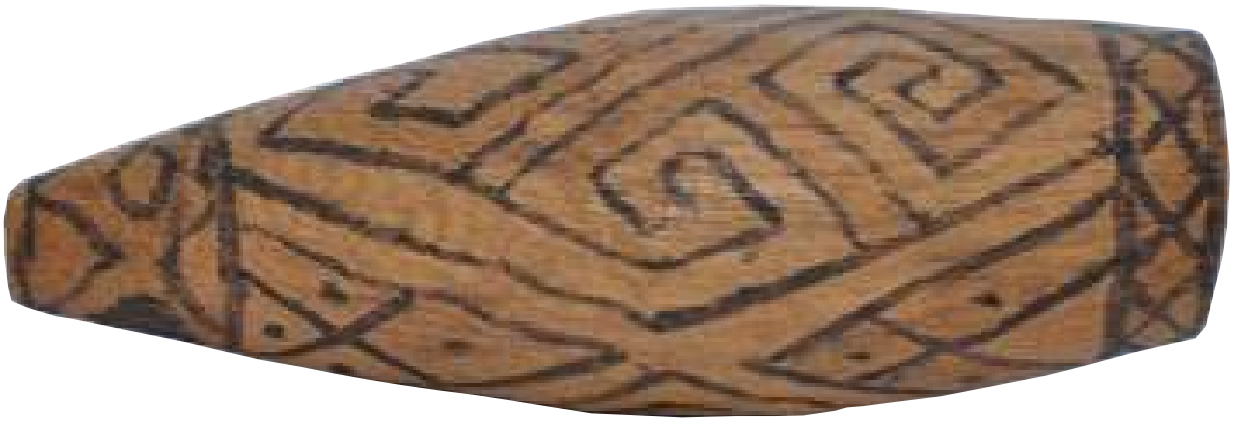

plus important que le thème explicite représenté. Lévi-Strauss, pour sa part, centre son analyse sur l'attention portée au style global du graphisme, sur la relation entre les lignes et sur celle entre le graphisme et son support.

L'utilisation du terme " abstrait » dans un contexte très différent de celui de l'art occidental ne va pas de soi et mérite d'être justifiée. Graphisme "géométrique " ou "aniconique " pourraient constituer des alternatives, mais ces termes représentent plutôt une conclusion qu'un point de départ pour l'analyse. Ainsi, le terme "aniconique" ne rend pas compte de la spécificité de systèmes graphiques qui peuvent receler des indices iconiques ${ }^{9}$. On n'a pas affaire non plus à une géométrisation d'images adoptant des figures comme modèle, puisque ces graphismes sont systématiquement associés à l'écriture plutôt qu'à la figuration. Les figures qui s'y cachent semblent être plutôt des effets secondaires d'une logique graphique propre, qui s'intéresse surtout aux relations entre les lignes. Les Amérindiens qui distinguent conceptuellement graphisme et figures tendent à associer les premiers à l'écriture des Blancs et les seconds au domaine des doubles, comme les reflets dans l'eau ou dans un miroir, les photos, les films et les « esprits ».

Le terme " abstrait » ouvre, en outre, des possibilités d'interprétation intéressantes, puisqu'il permet d'explorer les « connexions partielles » (Strathern 2004) entre l'art abstrait occidental et les "systèmes complexes de dessins" de l'Amazonie occidentale, en mettant en évidence les ressorts cognitifs de la pensée formelle. Si ces «traditions iconographiques non occidentales se fondent sur des principes très différents de l'imitation de la nature, le problème de la représentation "abstraite" n'est [...] ni propre à l'art moderne, ni réservé à la tradition occidentale " (Severi, à paraître). Un travail récent de Severi sur «l'enjeu de l'image dans la pensée de Claude Lévi-Strauss » aboutit à une analyse anthropologique de l'art occidental qui «vise à l'abstraction". Ma propre démarche part dans la direction inverse, celle d'une analyse anthropologique de l'art amérindien qui vise à l'abstraction.

"Pour l'art "spirituel" (terme pour lui synonyme d'“abstrait") que prône Kandindsky, le monde n'est donc plus le sujet de la représentation. Ce que l'artiste doit viser, délaissant les apparences, c'est l'acte mental que la perception du monde suppose. [...] Là où on ne veut voir qu'un vide, un manque de référence à la nature, on trouve une réflexion sur le regard. » (Severi, à paraître)

Ce que j'ai essayé de démontrer dans mes études sur le graphisme cashinahua illustre cette idée. Ce graphisme, en tant que technique d'éducation du regard, implique un engagement actif du spectateur avec l'espace cinétique créé par la relation entre les lignes. Comme les artistes occidentaux du mouvement abstractionniste, les artistes cashinahua et leurs congénères visent à travers leur jeu avec les lignes à produire une perception spatiale nouvelle qui ne se substitue pas à un espace préexistant, mais s'y ajoute. La transformation artistique de la perception spatiale consiste ainsi en une superposition plutôt qu'en une substitution ${ }^{10}$.
Fig. 6 Mortier à tabac cashinahua.

\section{PAGE SUIVANTE}

Fig.7 Panier kakan, utilisé par la femme du chef. L'homme le fabrique et la femme le peint d'un dessin (kene). Ici il s'agit du kene nommé xamantxin ou bawa. Photo Eliane Camargo.
- $\odot$

9. Pour une discussion critique de la distinction entre iconique et aniconique, voir Gell 1998.

10. Vilaça (s.d.) a noté un phénomène similaire en examinant les dessins figuratifs des Wari, où les vêtements laissaient transparaître le corps humain en dessous. 
III I I I I T

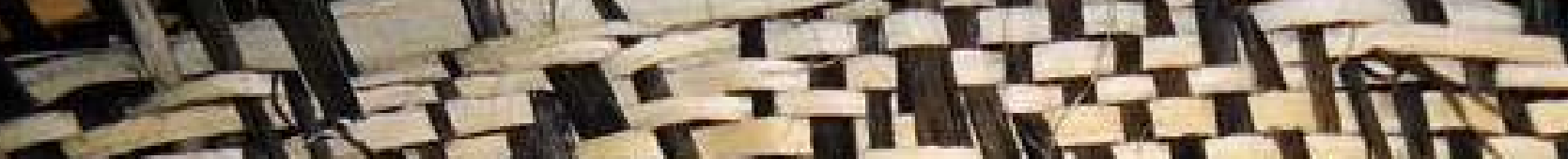
2 -

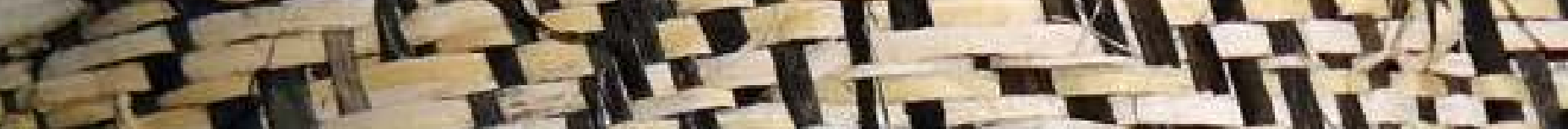
$4-11$ - 110 I

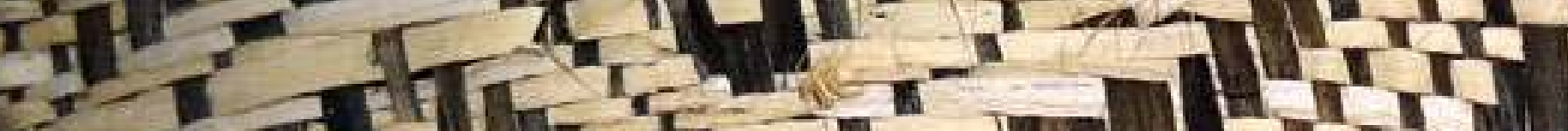
5. II 3. C.

$3=1$

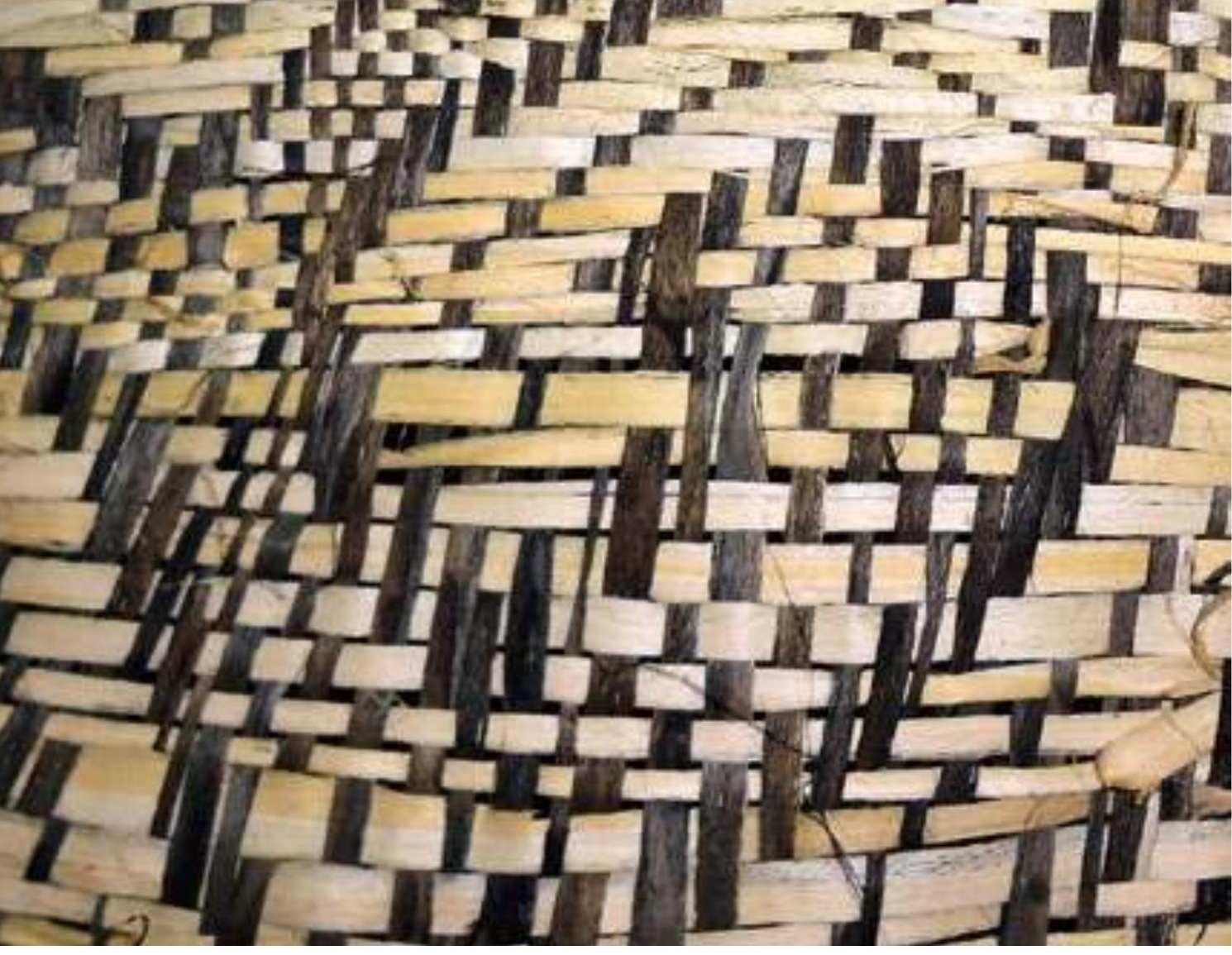


PIÈGES À VOIR, PIÈGES À PENSER

Le punctum, quand il devient excessivement visible, est le point de départ de la production d'un nouveau motif dans le même tissu. Un motif en génère un autre et aucune de ces images n'est image d'autre chose sinon de ce mouvement intra-imagétique. Ce dernier principe formel se retrouve dans les graphismes des Cashinahua, Wauja, Wayãpi, Shipibo et Sharanhua. Dans ce sens, on voit que le punctum relève ainsi d'une technologie de la transformation interne à la structure de l'image. Cette technologie a été également décrite par Barcelos Neto (2005) chez les Wauja, où les variations internes minimales sur les masques constituent une manière efficace de signaler la proximité de masques entre eux.

Un autre aspect formel est crucial dans la comparaison de ces graphismes amérindiens avec l'abstractionnisme. Il s'agit de l'interruption systématique du dessin, dès qu'il est devenu reconnaissable, de manière à suggérer sa continuité au-delà des limites du support. Ce dispositif stylistique révèle un élément important de la signification du dessin dans l'ontologie cashinahua : le rôle qu'il joue dans la transition entre perception imaginative et imagination perceptive (Lagrou 1998a, 1998b). Les Cashinahua produisent souvent aujourd'hui des tissus entièrement recouverts de dessins, surtout pour les commercialiser (fig. 8), tandis que dans les collections anciennes ${ }^{11}$ seule une bande étroite du tissu était dessinée. Si les dessins sont des pièges pour l'esprit de l'œil, seuls les tissus destinés aux Blancs constituent des pièges complets. Les "pièces" produites pour un usage interne se contentent d'amorcer des pistes.

Les Shipibo vont plus loin dans l'importance attribuée à la perception imaginative en disant que, pour qui possède la capacité de le voir, le corps humain lui-même est couvert de dessins. Cette peinture invisible fonctionne comme protection contre la maladie. Les chamanes peuvent visualiser les dessins sur la peau des patients quand ils sont sous l'influence de l'ayahuasca, et également voir à travers la peau, devenue transparente (Gebhart-Sayer 1986).

Chez les Asurini aussi, le dessin suggère sa continuité au-delà des frontières du support. Dawson (1975) et Müller (1990 : 232) qualifient cet aspect de «fenêtre vers l'infini ». Les Cashinahua mettent en valeur cet effet d'indépendance du graphisme par rapport au support en plaçant le motif de manière oblique sur ledit support. Une autre façon de jouer de la tension entre visibilité et invisibilité consiste à tisser ou tresser des motifs sans utiliser de couleurs contrastées. Le umin kene, nom donné à cette technique utilisée pour les hamacs, est très valorisé par les Cashinahua pour la difficulté posée autant par sa fabrication que par sa perception. Les paniers sont tressés selon la même technique. Quand ils sont neufs, on peut dessiner le contour des motifs avec du roucou, mais cette aide à la perception est vite effacée par le temps.

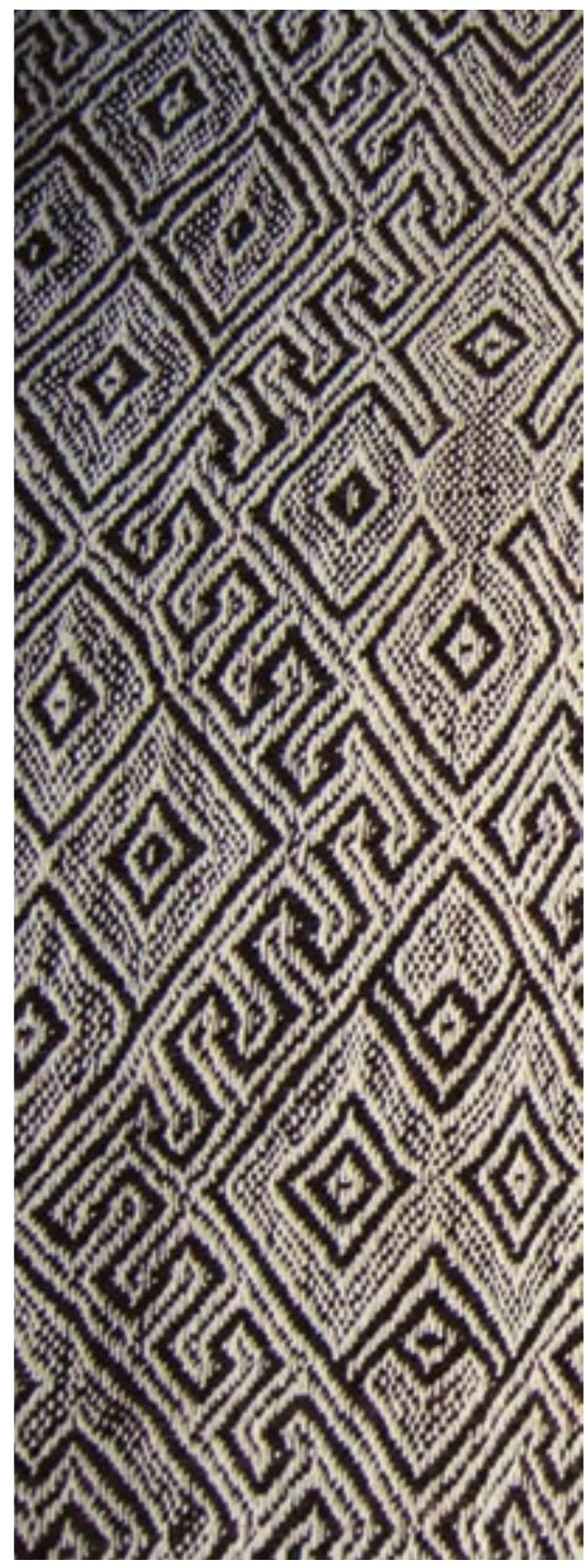

11. Celles

11. Celles de Schultz, au musée d'Archéologie et d'Ethnologie de l'université de São Paulo, et de Kensinger (voir Dwyer 1975). 


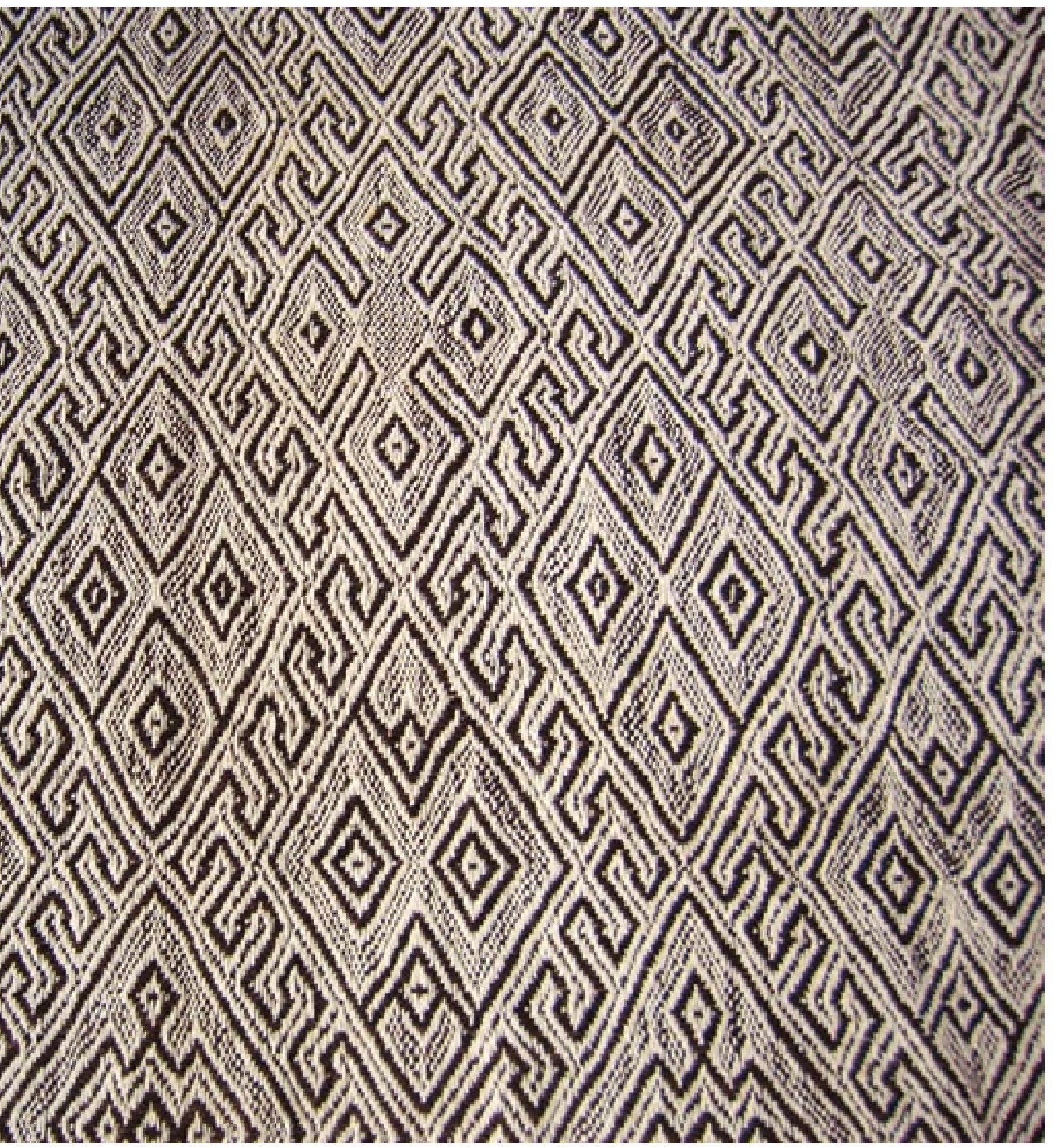

Fig 8 Hamac cashinahua aux motifs dunan kene (dessin de l'anaconda) et hua kene (dessin de fleur). 


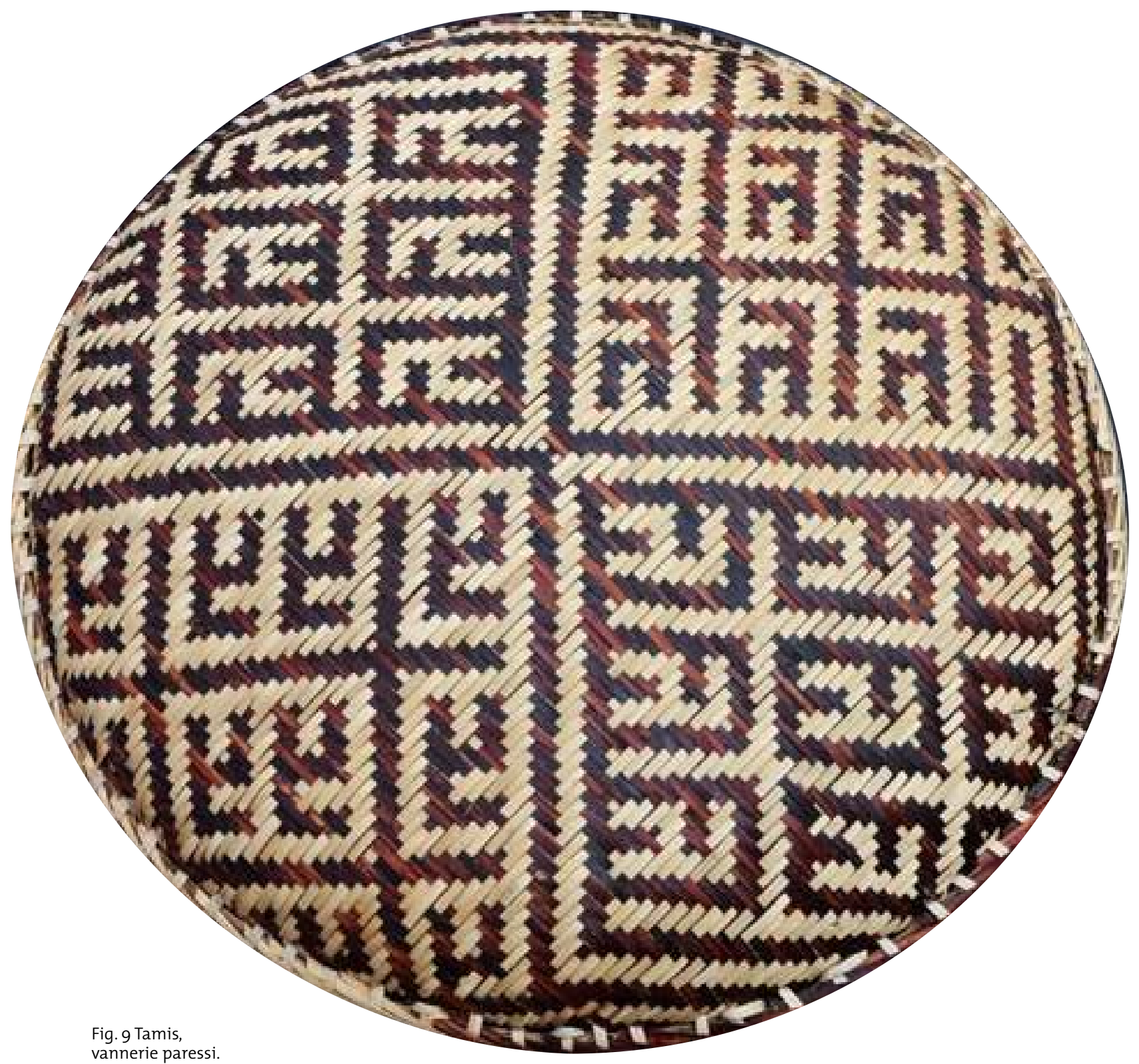




\section{La chimère}

Qu'est-ce qu'une chimère? Le terme peut renvoyer à deux genres de phénomènes qu'il est important de distinguer. Severi prend comme point de départ pour sa définition la chimère hopi, analysée par Warburg. Celui-ci a trouvé dans les dessins d'enfants hopi des images de serpent-foudre, et dans la poterie, des images d'oiseau-serpent. Pour généraliser le genre d'opérations mentales impliquées, Severi mobilise d'abord une définition englobante du phénomène chimérique, "comme l'association en une seule image de traits hétérogènes provenant d'êtres différents. La chimère grecque, corps monstrueux associant serpent, lion et oiseau, en est un exemple bien connu " (Severi 2007 : 69-70).

Ce genre d'image, en tant qu'être composite où la différence entre les parties composantes est maintenue, est considéré par Philippe Descola comme un procédé de figuration commun dans les pensées analogistes (2010:22-24). Severi note néanmoins une différence cruciale pour notre argument entre la chimère grecque et la chimère hopi, différence qui nous met sur le chemin de la transformation propre à l'art des Amérindiens :

"Toutefois, il est clair que la chimère hopi offre à l'œil beaucoup moins de détails, elle simplifie sa structure. C'est sur la base de cette conventionnalisation [...] qu'elle déclenche une projection de la part de l'œil, qui fait ainsi surgir activement son image en la complétant. Il faut ici noter deux points : non seulement l'image se partage en deux parties, l'une matérialisée et l'autre mentale, mais l'espace dans lequel elle est complétée est entièrement mental. Dans une poterie hopi, seul le support du vase, plat ou convexe, fournit à l'œil une indication de l'espace dans lequel situer l'image. Toute autre indication est le fruit d'un acte de regard, fait à la fois de projection et d'association. Nous découvrons là une différence cruciale entre chimère grecque et chimère hopi. Ni leur rapport à l'invisible, ni leur manière d'engendrer un espace mental ne sont de même type. En tant que résultat d'une conventionnalisation iconographique, la chimère hopi est donc un ensemble d'indices visuels où ce qui est donné à voir appelle nécessairement l'interprétation de l'implicite. Cette partie invisible de l'image se trouve entièrement engendrée à partir d'indices donnés dans un espace mental. Il existe un principe qui sous-entend la structure de ces images-chimères, où l'association de traits hétérogènes implique nécessairement une articulation particulière entre le visible et l'invisible. Cette structure par indices, où la condensation de l'image en quelques traits essentiels suppose toujours l'interprétation de la forme par projection, et donc par remplissage des parties manquantes, a une conséquence importante : elle confère à l'image une saillance particulière qui la distingue d'autres phénomènes visuels." (Severi 2007: 70, je souligne)

Au-delà de la capacité mnémonique de ce genre d'image, mise en avant par Severi, ce qui m'intéresse ici est la relation entre une cosmologie spécifique qui met la transformation au centre de ses préoccupations et un style graphique qui joue constamment sur la tension entre ce qui est montré et ce qui n'est pas montré. Le cas du graphisme cashinahua dialogue évidemment plus avec la chimère hopi qu'avec la chimère grecque. Le caractère chimérique des graphismes amérindiens renvoie au mouvement de transformation entre les corps plutôt qu'au caractère composite des êtres. De la même manière, pour indiquer une intériorité partagée et pour montrer la possibilité de passage de la figure humaine à la figure d'un ours ou vice versa, Descola (2010) montre une image à mi-chemin entre face humaine et face d'ours. Le statut ambivalent et double de la tête réduite des captifs jivaro suit la même logique : 
-

12. Pour les Yaminahua, voir Townsley (1993 : 449-468).

13. Il semble que le même phénomène puisse être trouvé dans d'autres groupes de langue tupi étroitement apparentés aux Asurini (Silva Fabiola, communication personnelle).
"[...] la tête est une "vraie personne" inventée, issue du prélèvement d'un visage existant apposé sur un être rituellement constitué. D'où les caractéristiques formelles de ces objets, qui portent toujours la marque d'une disposition à la prédation (indice de leur position d'ennemi par rapport au groupe des preneurs de tête), une ornementation de plumes et d'autres parures signalant la virtualité d'une identité tribale, enfin une enveloppe ou une apparence corporelle synthétisant des identités contradictoires, par exemple celle de membre du collectif des morts et en même temps d'une société d'humains, d'homme en même temps que de femme, d'adulte en même temps que d'enfant. » (Taylor $2010:$ 47-48)

\section{De l'abstraction à la figuration}

Mon hypothèse est qu'il existe des continuités chez les Amérindiens, surtout en Amazonie, entre les modes de figuration d'une part et les graphismes de l'autre : dans le cadre de la cosmologie transformationnelle amazonienne, la relation entre graphisme et figure est elle aussi une relation de transformabilité, le graphisme étant un chemin optique pour la visualisation d'images virtuelles. C'est pour cette raison que les dessins ne représentent pas autre chose que l'acte de voir lui-même, qui se focalise sur une superficie pour la dépasser. "Le dessin est un chemin ", disent littéralement les Cashinahua ainsi que leurs voisins pano ${ }^{12}$, une "porte d'entrée " : il se réfère à d'autres images, toutes également en mouvement. Les exégèses explicites fournies par les Cashinahua sur leurs systèmes visuels me permettent de formuler cette hypothèse du dessin comme passage vers la figuration virtuelle, mais je pense que l'on peut étendre celle-ci à d'autres systèmes graphiques.

Celui des Asurini en offre une illustration paradigmatique. Au lieu de distinguer conceptuellement graphisme et figuration, comme le font les Cashinahua, les Piro, les Wayana, les Wauja et tant d'autres, les Asurini perçoivent la figuration de l'invisible comme partie intégrante de l'unité minimale de signification qu'est le motif tayngava (un angle de 90 degrés), présent dans la plupart des peintures corporelles. Toute abstraction pointe donc ici déjà vers la figuration $^{13}$. L'image affleure entre les lignes du graphisme qui adhère au corps, mais il ne devient véritablement figure que lorsqu'on fabrique une effigie, une poupée schématique qui représente l'âme dans les rituels chamaniques.

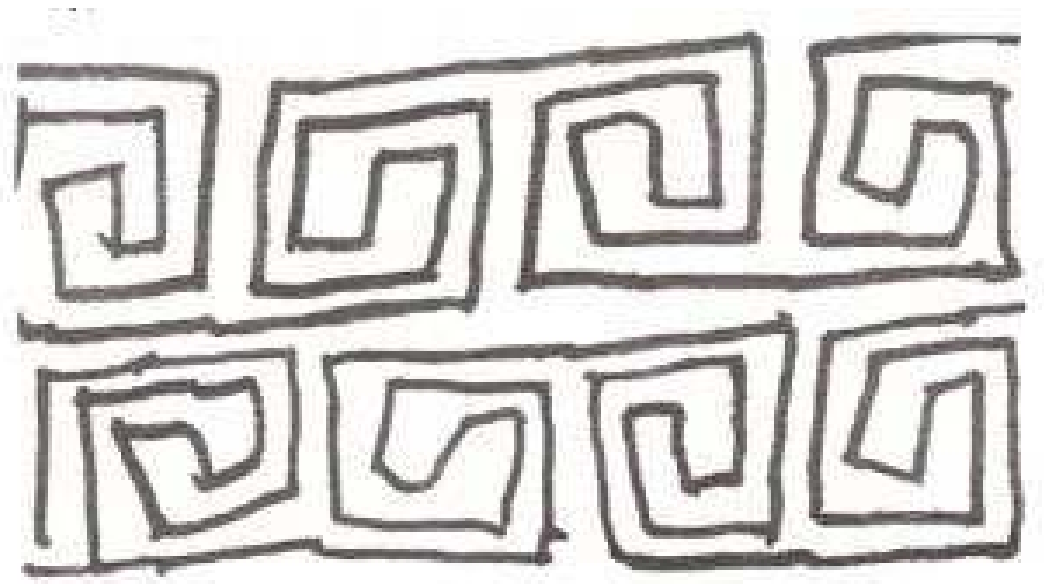




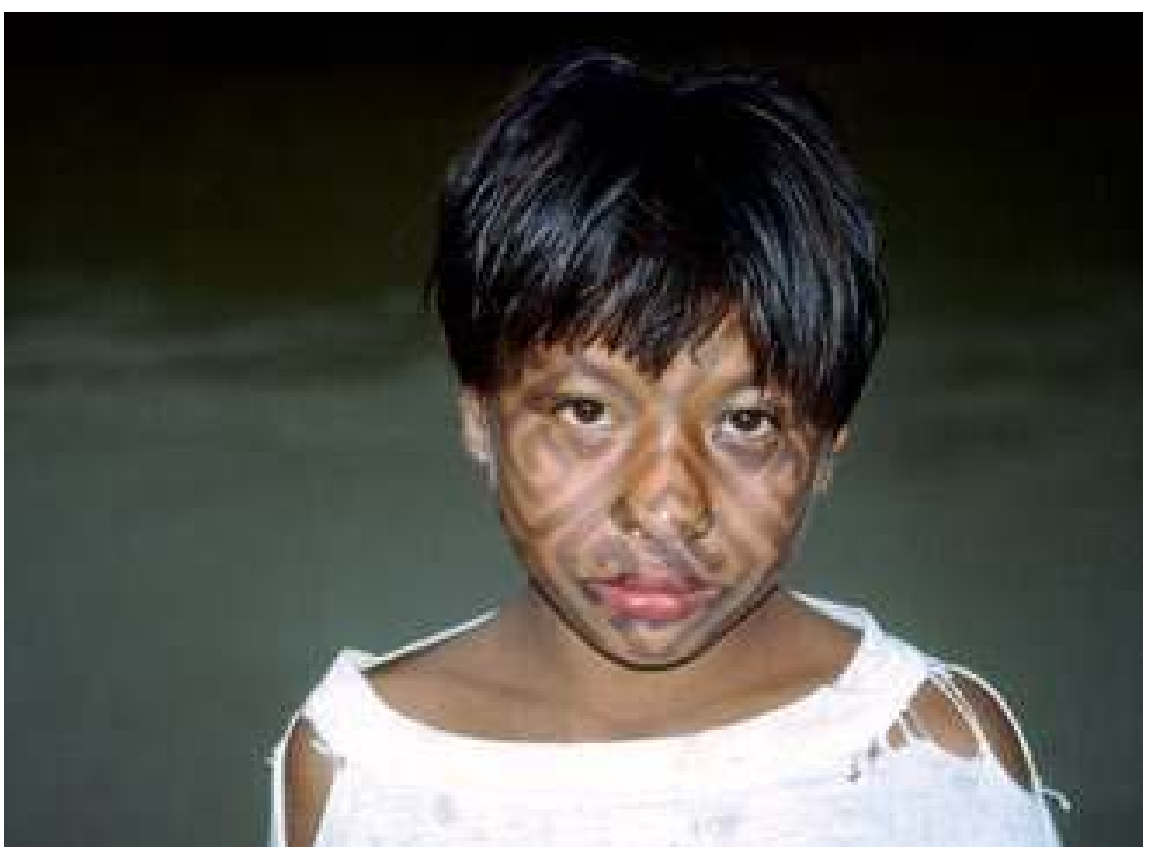

Le graphisme se transforme ici clairement en figuration virtuelle, qui repose sur la perception d'indices minces permettant de compléter mentalement l'image. Ce mouvement n'est cependant accessible qu'aux personnes qui ont été formées à ce cadre de référence. Les Asurini perçoivent le motif tayngava comme un passage vers la perception des images virtuelles des «esprits " a'anga :

"Dans la mythologie, les héros créateurs sont humains; les animaux ont la forme humaine et les esprits actuels sont anthropomorphes. Les Asurini disent de ces êtres qu'ils étaient tous avá (des personnes) dans le passé mythique. L'homme se trouve, ainsi, au centre de la pensée asurini; l'homme est l'image de l'être Tayngava. " (Müller $1990: 250)$

Les Cashinahua, pour leur part, n'ont pas de motif qui pointe explicitement vers la perception virtuelle de formes humaines, mais les figures ci-dessous montrent que cette idée ne leur est pas étrangère. Il est possible que le motif nawan kene (dessin de l'étranger), composé également d'un angle de 90 degrés, puisse être interprété dans ce sens (fig. 10).

Les Cashinahua ont plusieurs noms de motifs et de dessins, mais ils insistent sur le fait que leur dessin est « un seul " (habiaski), qu'il sert à signaler ce que les "êtres à dessin " (keneya) ont en commun, non ce qui les différencie. Les «êtres à dessin " possèdent aussi un yuxin fort, ce sont les maîtres de la transformation, comme le "jaguar à dessin " (inu keneya), l'anaconda (yube) et le boa (sidika), certaines plantes utilisées pour modifier la perception ${ }^{14}$, comme le bawe et le mani pei keneya, les papillons à dessin, la tortue, et d'autres. Tous sont des animaux emblématiques de l'art de la transformation (Lagrou 1995). Les Cashinahua insistent sur le fait que le nom des motifs n'est qu'un nom et que le système graphique (kene, mot utilisé pour traduire «écriture ") est «la langue des esprits " (yuxinin hantxaki). D'autre part, puisque chaque «être à dessin " possède son dessin, ainsi qu'un nom et un chant, ces trois indices fonctionnent comme des opérateurs mnémotechniques : on peut appeler ces êtres par leur nom, leur chant et leur dessin (Lagrou 1998a, 1998b, 2007a).
Fig. 11 Enfant soumis au rite de passage nixpupima. Photo Els Lagrou.
-

14. Des plantes qui changent la perspective se trouvent dans toute l'aire pano, chez les Shipibo-Conibo (Colpron 2004 ; Belaunde 2009), chez les Yaminahua, Katuquina, Yawanawa (cf. Lagrou 2007a), et Sharanahua (Déléage 2009). 


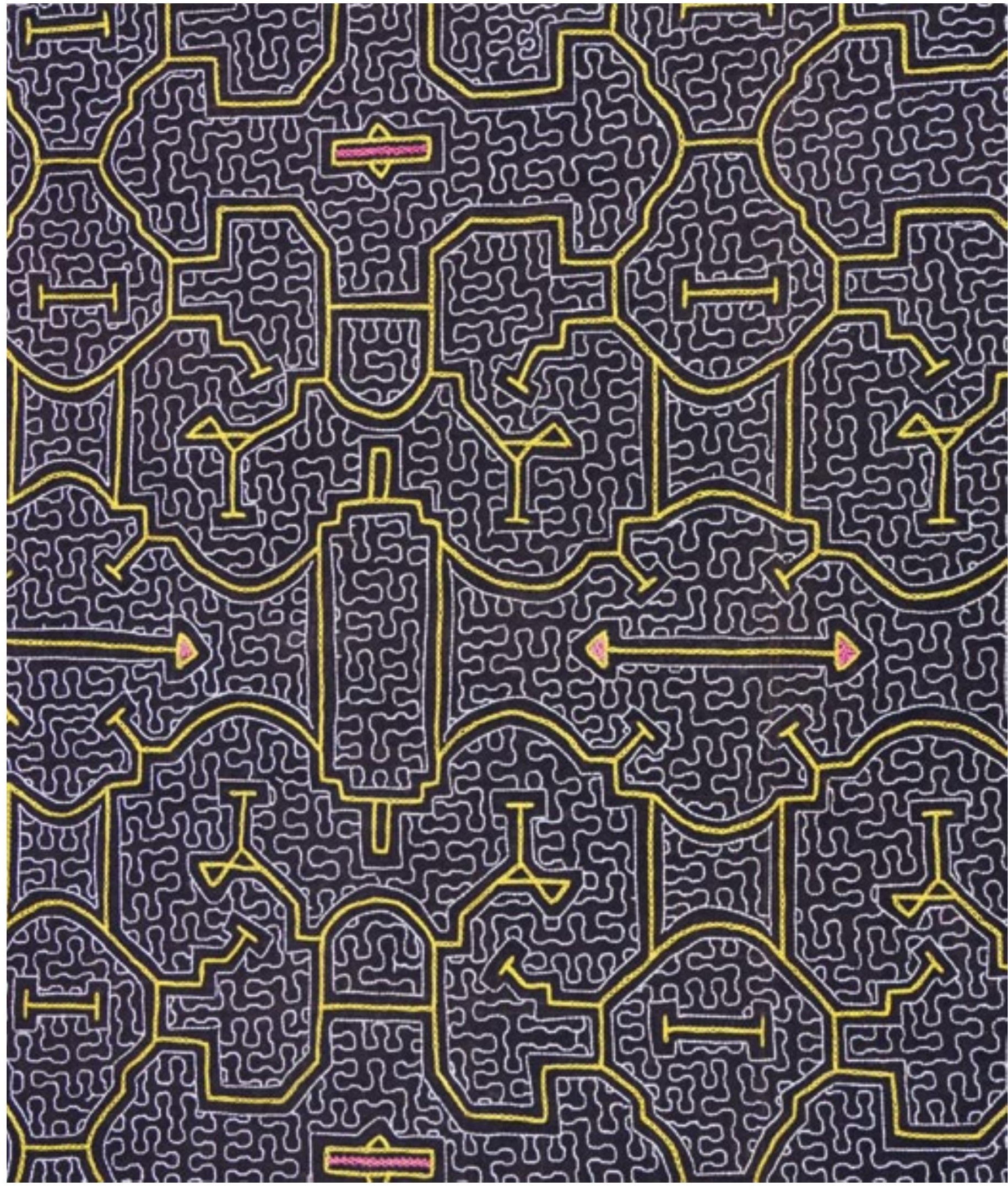


Je me suis rendu compte de l'importance perceptuelle de la différence entre dessin graphique (kene) et figure (dami) quand j'ai vu la réaction à la première peinture faciale que j'ai exécutée, après m'être longuement exercée sur papier. La jeune femme que j'avais peinte, après s'être regardée dans le miroir, ne cachait pas son insatisfaction et s'est empressée d'effacer ce dessin mal fait. Je péchais visiblement par la technique de composition, en ne respectant pas la focalisation perceptive qui y préside normalement. J'avais en effet visualisé mentalement le motif final que je voulais obtenir. Or, là résidait le problème : en dessinant la figure géométrique, j'avais perdu de vue la relation entre les lignes (elles devaient se toucher, devaient maintenir la même distance entre elles), et la relation entre les lignes et la surface irrégulière du visage. La dynamique propre au graphisme s'en trouvait perdue, en particulier sa capacité à créer une alternance entre deux perceptions, selon ce qu'on identifie comme figure et comme fond : mon dessin ne donnait à voir qu'une figure stable sur un fond stable.

Le dessin résultant était mal fait et risquait de devenir une figure (dami) au lieu d'un dessin (kene) propre, un vrai dessin obéissant aux règles de la composition stylistique. Peindre des dessins sur la peau n'équivalait visiblement pas à la projection d'une diapositive sur une superficie quelconque. C'est à partir de ce moment que j'ai commencé à observer le processus de composition d'un dessin par les Cashinahua, processus que j'ai reconstitué dans la figure suivante. J'ai alors compris que si l'on se concentre sur la relation entre les lignes, on obtient un dessin que l'on visualise seulement à la fin et qui répond aux exigences stylistiques de l'alternance entre figure et fond. Il en résulte une perception labyrinthique qui ne révèle pas immédiatement les figures possibles.

Déléage a constaté un procédé similaire chez les Sharanahua, voisins pano des Cashinahua :

«Plutôt que de dessiner une croix par l'intersection, en leur milieu, de deux traits identiques, les Sharanahua tracent d'abord un chevron auquel ils accolent par la suite son symétrique en reflet [...]. Le carré, quant à lui, n'est pas réalisé à l'aide d'un

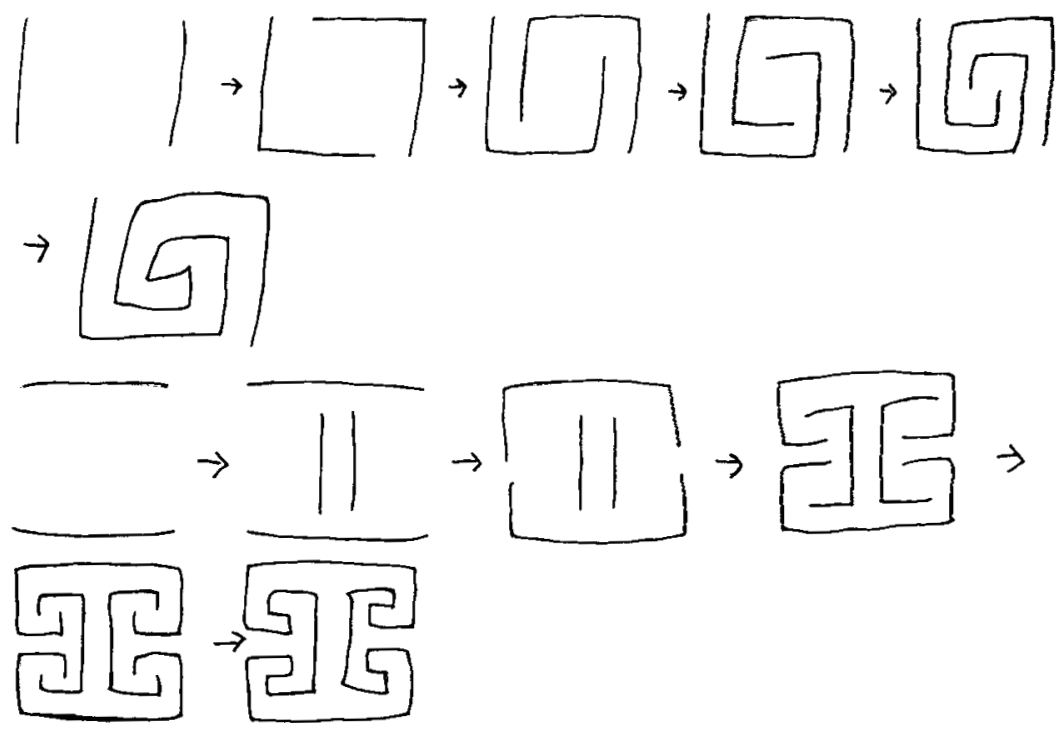

Fig. 13 Dessin reproduisant la constitution progressive de deux motifs cashinahua, le isu meken (main de singe) et le xamanti (Lagrou 1991 : 142 ; 2007a). 


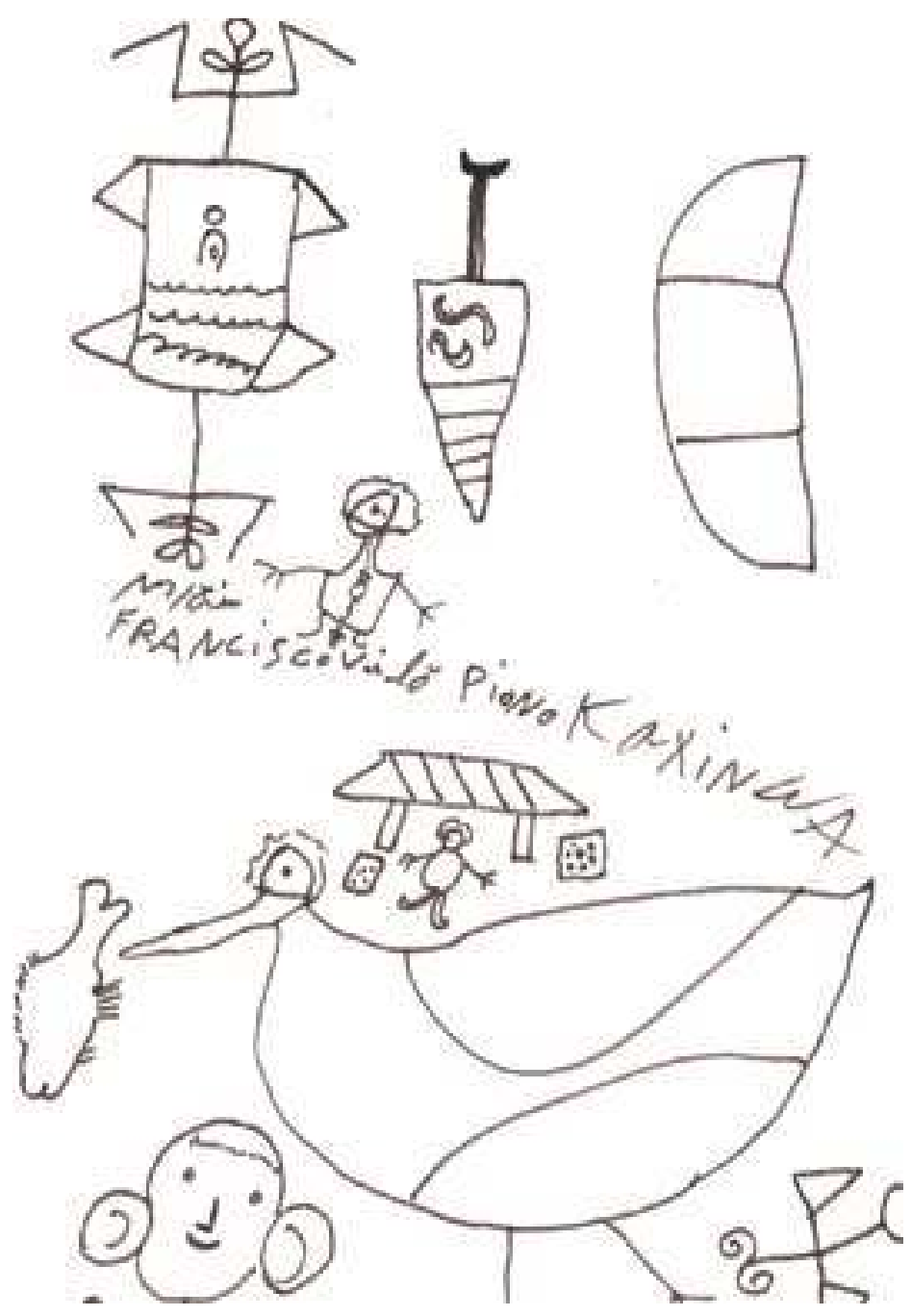

Fig. 14 Dessin sur papier par Francisco Filó Kaxinawa qui suggère le passage du kene (graphisme) au dami (figure).

trait continu, mais résulte de l'opération inverse de symétrie appliqué à ces mêmes chevrons [...]. Une fois isolé, ce simple motif, pour lequel les Sharanahua n'ont pas de mot particulier, permet de reconstruire tous les motifs de leur répertoire graphique. » (Déléage 2007: 100)

Ayant ainsi identifié les éléments de base non nommés, l'auteur mentionne les opérations graphiques qui «mettent en jeu les lois bien connues de la composition au sein desquelles on reconnaîtra la symétrie (en miroir ou inversée), la translation et la rotation" (ibid. : 101). Mais regardons de plus près la constitution de ces "simples motifs", le carré et la croix. Si l'on compare les figures cashinahua et sharanahua, on constate que leur composition suit la même logique, celle de combiner des lignes sans visualiser une figure, obéissant à la dynamique de la «représentation dédoublée " (Boas 1955 [1928]; Lévi-Strauss 1955, 1958). Chez les Sharanhua et Yaminahua, contrairement aux Cashinahua, les dessins ne couvrent pas la face entière. Ils combinent des dessins aux contours délimités, placés sur les joues, et des graphismes qui couvrent une partie du visage, le menton 


\section{PUNTURA FACAL EM CRIANGES}

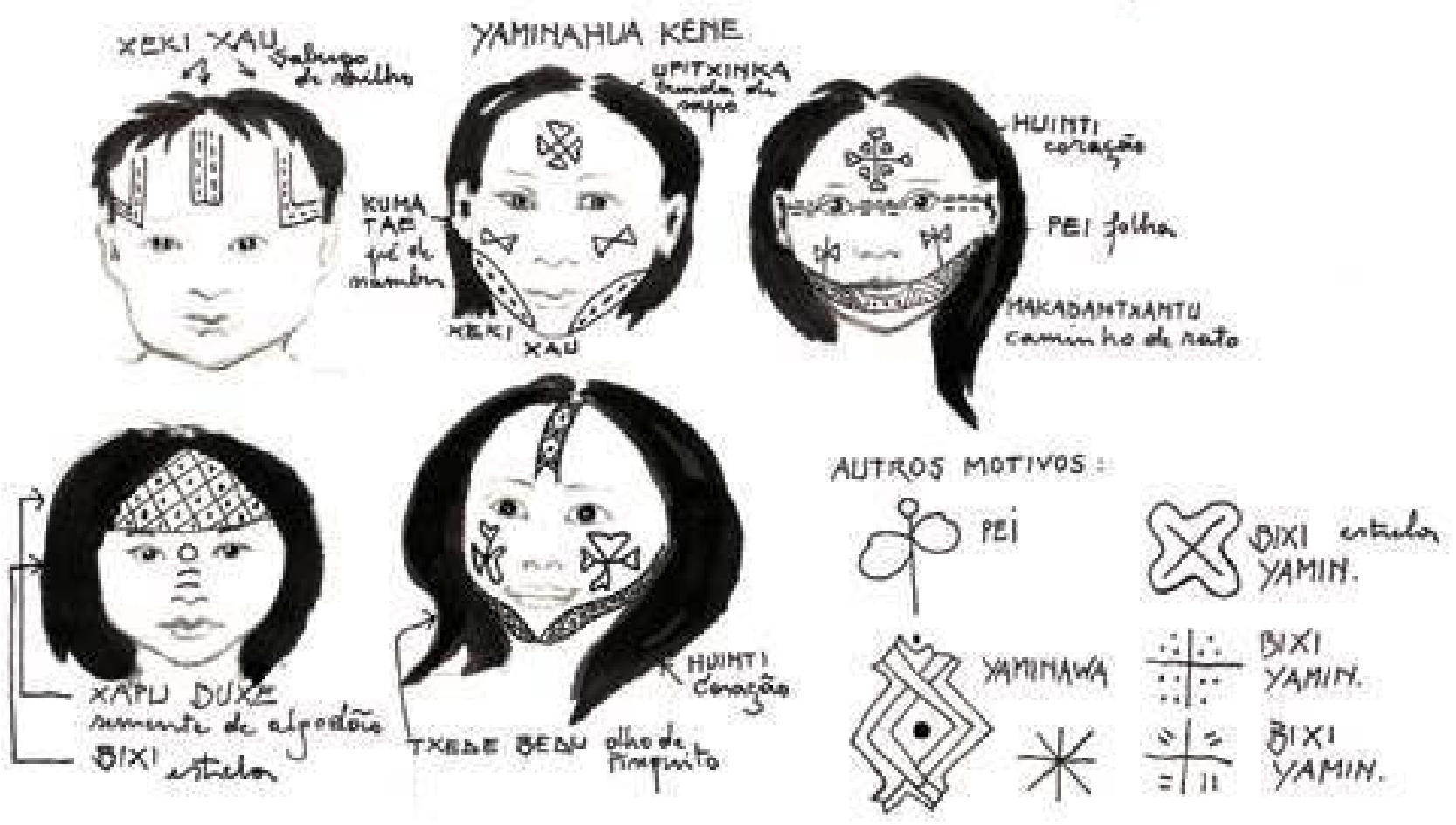

Fig. 15 Yaminahua kene, peinture faciale des enfants. Dessin Els Lagrou.

et les maxillaires, des coins de la bouche jusqu'aux oreilles. Ce que suggère la logique de composition identifiée par Déléage, néanmoins, c'est que ces motifs cachent une même logique combinatoire des lignes qui sont interrompues dès que le motif devient reconnaissable. Cette interprétation révèle une grande continuité formelle entre les différents styles de graphisme pano.

Pour Lévi-Strauss, l'artificialité de l'ordre spatial, imposé par le graphisme sur la géographie anatomique du visage, rendait visible la double identité de son porteur, en même temps qu'il transformait profondément son apparence. Loin de la logique du maquillage qui sert à renforcer la physionomie naturelle du visage et à cacher ses imperfections, on se trouverait donc ici dans la logique du masque - mais d'un masque qui, au lieu de cacher l'apparence originale, vise à sa transformation ambivalente, en laissant transparaître la dualité constitutive de la personne. Le graphisme crée ainsi, pourrait-on dire, une «identité complexe » ou une «chimère ".

La tension dynamique entre dessin et visage, entre graphisme et support, peut cependant renvoyer à une dualité autre que celle du masque, si l'on considère que le graphisme aide en fait à constituer une peau, l'interface entre un contenu et un contenant. Les lignes tracées ne laissent pas intact leur support, elles le transforment. Quand les Cashinahua, Shipibo et Caduveo se peignent le visage, ils le sectionnent selon des axes spatiaux propres à la logique stylistique en question : horizontalement, verticalement ou en diagonale. Les lignes tirées au genipa vont 
graduellement emplir l'espace démarqué à la manière d'un filigrane, d'un labyrinthe. Le graphisme, en produisant une dynamique et une spatialité propres et internes aux relations entre les lignes constitutives du dessin, rend visible la perméabilité de la peau et sa proximité avec les matières tissées.

\section{Conclusion}

On a pu noter que les Cashinahua et leurs congénères mettent en œuvre plusieurs "techniques de médiation" pour concrétiser le passage entre différents points de vue. L'étude de la "chimère abstraite " peut nous aider à regarder avec plus d'attention les opérations mentales spécifiques qui permettent le passage entre différents modes de connaissance. La condensation rituelle engendre une "réalité complexe", différente de la réalité quotidienne (Houseman et Severi 1994). La pratique esthétique engendre également des réalités complexes qu'on ne trouve pas dans la réalité telle qu'elle est observable. Dans ce sens, le rituel, l'art (en tant que production d'images virtuelles et matérielles), et le chamanisme sont autant de «techniques de médiation " qui montrent une réalité non observable à l'œil nu.

Dans la combinaison d'un anthropomorphisme discret des objets avec le graphisme en filigrane qui les recouvre, on trouve une application de la centralité de la transformation et des métamorphoses dans le monde amazonien animiste et perspectiviste. Le concept de chimère proposé par Severi nous permet d'élaborer une approche précise des techniques formelles et cognitives qui sont mises à l'œuvre dans le graphisme des « systèmes complexes de dessin » en tant que forme active et instrument dans l'apprentissage du passage de la perception visuelle à la perception virtuelle. Le graphisme appliqué sur les corps est une technique de visualisation des processus de transformation, qui permet de conceptualiser comment se produisent ces passages entre points de vue, comment des corps deviennent transparents, comment on passe d'une ligne à un visage.

Programa de Pós-Graduação em Sociologia e Antropologia, Instituto de Filosofia e Ciências Socias, Universidade Federal do Rio de Janeiro els@ifcs.ufrj.br

mots clés / keywords: Cashinahua // Cashinahua - chimères // chimeras • abstractionnisme // abstractionism • figuration // representation • perspectivisme // perspectivism. 


\section{Bibliographie}

\section{Barcelos Neto, Aristóteles}

2008 Apapaatai. Rituais de Máscaras no Alto Xingu. São Paulo, EDUSP-FAPESP.

\section{Bateson, Gregory}

1977 Vers une écologie de l'esprit.

Paris, Seuil.

\section{Belaunde, Luisa Elvira}

2009 Kené. Arte, ciencia y tradición en diseño. Lima, Instituto Nacional de Cultura.

\section{Beysen, Peter}

2008 Kitarentse: Pessoa, arte e estilo de vida ashaninka (do Oeste Amazônico). Rio de Janeiro, PPGSA-IFCS-UFRJ, thèse de doctorat.

\section{BoAs, Franz}

1928 [1955] Primitive Art. New York, Dover publications.

\section{CAMARgo, Eliane}

1995 Léxico Caxinauá-Português. Paris, Association d'ethnolinguistique amérindienne [«Chantiers Amerindia »).

\section{Capistrano de Abreu, João}

1941 [1914] Rã-txa hu-ni-kuiff: A Língua dos Caxinauás do Rio Ibuaçú. Rio de Janeiro, Leuzinger-Livraria Briguiet.

Dawson, Alice

1975 « Graphic Art and Design of the Cashinahua », in Jane Powell Dwyer (éd.), The Cashinahua of Eastern Peru. Philadelphia, Haffenreffer Museum of Anthropology : 131-149.

DéléAge, Pierre

2007 «Les répertoires graphiques amazoniens », Journal de la Société des Américanistes 93[1] : 97-126.

Descola, Philippe

2005 Par-delà nature et culture. Paris, Gallimard.

Descola, Philippe (dir.)

2010 La Fabrique des images. Visions du monde et formes de la représentation. Paris, Somogy-musée du quai Branly.

FrANCHeTto, Bruna (éd.)

2003 Ikú ügühütu higei. Arte gráfica dos povos karib do alto Xingu. Rio de Janeiro, Museo do Indio-FUNAI.

\section{Galiois Tilkin, Dominique}

2002 Kusiwa: pintura corporal e arte gráfica waiãpi. Rio de Janeiro, Museu do Indio-FUNAICTI-NHI-USP.

\section{GeBhart-Sayer, Angélica}

1986 «Una terapia estética: Ios diseños visionarios del ayahuasca entre los ShipiboConibo », America Indígena 46 : 189-218.

\section{GeLl, Alfred}

1993 Wrapping in Images. Tattooing in Polinesia. Oxford, Clarendon Press.

1998 Art and Agency. An Anthropological Theory. Oxford, Oxford University Press.

\section{Gow, Peter}

1989 «Visual Compulsion: design and image in Western Amazonian art », Revindi. Revista indigenista Americana 2 : 19-32. 1999 « Piro Designs: painting as meaningful action in Amazonian lived world », Journal of the Royal Anthropological Institute 5 : 229-246.

2001 An Amazonian Myth and its History. Oxford, Oxford University Press.

\section{Guss, David}

1989 To Weave and Sing. Art, Symbol and Narrative in the South American Rain Forest. Berkeley-Los Angeles, University of California Press.

\section{Houseman, Michael et Severi, Carlo} 1994 Naven ou le donner à voir. Essai d'interprétation de l'action rituelle Paris, CNRS Éditions-Éditions de la MSH.

\section{Iluius, Bruno}

1987 Ani Shinan : Schamanismus bei den Shipibo-Conibo (Ost-Peru). Münster, Verlag S \& $\mathrm{F}$.

\section{INGOLD, Tim}

2007 Lines. A Brief History. London, Routledge.

\section{KeIFenheIM, Barbara}

1996 «Snake Spirit and Pattern Art. Ornamental Visual Experience among the Cashinahua Indians of Eastern Peru », ms.

1998 Untersuchungen zu den Wechelbeziehungen von Blick und Bild. Die Kashinawa-Indianer und ihre Ornamentik [Ost-Peru]. Berlin, Freie Universität, thèse de doctorat.

\section{LAGROU, Els}

1991 Uma Etnografia da Cultura Kaxinawá: entre a Cobra e o Inca. Florianópolis, UFSC, mémoire de master.

1995 «Compulsão Visual. Resenha do artigo "Visual compulsion" de Peter Gow », Antropologia em primeira mão 9.

1996 «Xamanismo e Representação entre os Kaxinawá », in E. J. Langdon (org.), Xamanismo no Brasil. Novas Perspectivas. Florianópolis, Editora UFSC : 197-231.

1998a Cashinahua Cosmovision: a perspectival approach to identity and alterity. Andrews, Université de St. Andrews, thèse de doctorat. 1998b Caminhos, duplos e corpos. Uma abordagem perspectivista da identidade e alteridade entre os Kaxinawa. São Paulo, USP, thèse de doctorat. 2002a « 0 que nos diz a arte kaxinawa sobre a relação entre identidade alteridade ? », Mana 8 : 29-62.

$2002 \mathrm{~b} \ll$ Kenan, the ritual stool: a reduced model of the Cashinhua person during the Nixpupima rite of passage », in T. Myers et M. S. Cipolletti [éd.], Artifacts and society in Amazonia / Artefatos y sociedad en Amazonia. Bonn : Bonner amerikanische studien : 95-113.

2005 « L’Art des Indiens du Brésil. Altérité, "authenticité" et "pouvoir actif" », in L. Donisete (éd.), Brésil indien. Les arts des Amérindiens du Brésil. Paris, Éditions de la Réunion des musées nationaux: 68-81.

2007a A fluidez da forma: arte, alteridade e agência em uma sociedade amazônica (Kaxinawa, Acre). Rio de Janeiro, Topbooks. $2007 \mathrm{~b} \ll$ Social metaphors of sociality and personhood in Cashinahua ritual song », in U. Demmer \& M. Gaenszle [éd.], The Power of Discourse in Ritual Performance. Rhetoric and Poetics. Berlin-London-Vienna : Lit Verlag [ «Intercultural Studies on Ritual, Play and Theatre $10 \gg]$ : 174-200.

2009a Arte indígena no Brasil: agência, alteridade e relação. Belo Horizonte, Editora C/Arte.

2009b « The Crystllized Memory of Artifacts: A Reflection on Agency and Alterity in Cashinahua Image-Making », in Fernando Santos-Granero (éd.), The Occult Life of things. 
Native Amazonian Theories of materiality and Personhood. Tucson, University of Arizona Press : $192-213$.

\section{LÉVI-STrauss, Claude}

1955 Tristes Tropiques. Paris, Plon.

1958 Anthropologie Structurale. Paris, Plon.

1964 La Pensée sauvage. Paris, Plon.

1991 Histoire de Lynx. Paris, Plon.

\section{Montag, Susan}

1981 Diccionario Cashinahua. YarinacochaLima, Ministério de Educacion-Instituto Lingüístico de verano.

\section{Müller, Regina Pólo}

1990 Os Asuriní do Xingu. História e Arte. Campinas, Unicamp.

Roe, Peter

1975 «Comparing Panoan Design Systems through Componential Analysis », ms.

\section{SeVeri, Carlo}

2007 Le Principe de la chimère. Une anthropologie de la mémoire. Paris, éditions rue d'Ulm-musée du quai Branly [« AEsthetica 》].

2009 « L'univers des arts de la mémoire. Anthropologie d'un artefact mental » in Annales HSS 64(2) : 463-493.

À paraître « L'idée, la série et la forme. Enjeux de l'image dans la pensée de Claude Lévi-Strauss ».

\section{Strathern, Marilyn}

2004 Partial Connections. Oxford, Altamira Press.

\section{TAYLOR, Anne-Christine}

2003 « Les masques de la mémoire. Essai sur la fonction des peintures corporelles jivaro ». L'Homme 165 : 223-248.

2010 « Voir comme un autre : figurations amazoniennes de l'âme et des corps », in Philippe Descola (dir.], La Fabrique des images. Visions du monde et formes de la représentation. Paris, musée du quai Branly-Somogy : 41-50.

\section{Townsley, Graham}

1993 «Song Paths. The Ways and Means of Yaminahua Shamanic Knowledge », L'Homme 126-128 : 449-468.

\section{Velthem, Lúcia Hussak van}

20030 Belo é a Fera. A estética da produção e da predação entre os Wayana.

Lisbonne, Museu Nacional de Etnologia.

VidAL, Lux (dir.)

1992 Grafismo indígena. São Paulo, Studio Nobel-Fapesp-Edusp.

\section{Viveiros de Castro, Eduardo} 1996 « Os pronomes cosmológicos e o perspectivismo ameríndio », Mana 2(2) : 115-144.

\section{Résumé / Abstract}

Els Lagrou, Le graphisme sur les corps amérindiens. Des chimères abstraites ? - Je propose de montrer dans cet article comment plusieurs techniques formelles utilisées dans le graphisme des Indiens cashinahua et d'autres «peuples à dessin » peuvent être vues comme des techniques perspectivistes, c'est-à-dire des techniques qui aident à visualiser la potentialité de transformation des phénomènes perçus. Si l'analyse des circonstances dans lesquelles ce processus peut se produire a été amplement réalisée dans les ethnographies de la région, en revanche les techniques de cadrage visuelles qu'il implique n'ont pas été suffisamment mises en lumière. C'est là qu'apparaît l'importance d'une étude des formes, d'une esthétique perspectiviste de la transformation. Au-delà du contexte perspectiviste et animiste dans lequel s'ancre cette recherche, j'explore la possibilité de considérer ces graphismes comme des «chimères abstraites».
Els Lagrou, Amerindian body art as abstract chimeras? - This article argues that several of the formal techniques used in the graphic designs of Cashinahua Indians and other so-called "graphic peoples" can be seen as perspectivist techniques-i.e. they assist in the visualisation of the transformative potential of perceived phenomena. Whilst there has been considerable ethnographic discussion of the circumstances in which such processes occur in the region, the visual framing techniques involved have been left largely unexplored. Whence the need for an analysis of the forms or the perspectivist aesthetic of transformation. As well as investigating the perspectivist and animist context of these processes, the article also considers the possibility of seeing such designs as "abstract chimeras". 